\title{
Recent Advances of Nanocarriers for Effective Delivery of Therapeutic Peptides
}

Mona Atabakhshi-Kashi ${ }^{1 \#}$, Marzieh Geranpayehvaghei ${ }^{1,2 \#}$, Yazhou Wang ${ }^{1}$, Hamidreza Akhbariyoon ${ }^{3}$, Mohammad Taleb ${ }^{1}$, Yinlong Zhang ${ }^{1}$, Khosro Khajeh $^{2,3^{*}}$, Guangjun $\mathrm{Nie}^{1,4 *}$
${ }^{I}$ CAS Key Laboratory for Biomedical Effects of Nanomaterials \& Nanosafety, CAS Center for Excellence in Nanoscience, National Center for Nanoscience and Technology, Beijing 100190, China
${ }^{2}$ Department of Nanobiotechnology, Faculty of Biological Sciences, Tarbiat Modares University, Tehran, Iran ${ }^{3}$ Department of Biochemistry, Faculty of Biological Sciences, Tarbiat Modares University, Tehran, Iran ${ }^{4}$ GBA Research Innovation Institute for Nanotechnology, Guangdong 510700, China

Submitted: April 27, 2020

Accepted: June 17, 2020

Published: June 23, 2020

Abstract

Precisely selective interactions of peptides with their unique binding partners represent an outstanding starting point for designing novel therapeutics. It is well established that peptides with a variety of critical physiological functions and specific mechanisms of action offer distinct advantages, including excellent safety and higher efficiency over traditional small molecule therapeutics. Certain intrinsic weaknesses of naturally occurring peptides such as negligible plasma half-life, low bioavailability, and potential immunogenicity have limited their administration as medicines. Nanotechnology has expanded several promising strategies to address the limitations associated with therapeutic peptides. This review aims to perform a state-of-the-art summary of the strategies that are actively used to develop efficient formulations of nanosystem based peptide medicines. We first focus on the recent advances and updates on peptide-based nanomedicines. Then we indicate how nanosystems improved the functionality of therapeutic peptides and what the future opportunities and challenges of developments in the field of therapeutic peptides are. Potential noninvasive delivery platforms for peptide incorporated nanoparticles through alternative administration routes are also discussed.

Keywords:

Therapeutic peptides; nanoparticles; peptide delivery; noninvasive administration; peptide encapsulation

\section{Rationale and Purpose}

One of the limitations of the therapeutic peptides is their inefficient delivery to the site of action. Since nanoparticles have significant potential as drug carriers, they can be used to overcome this limitation. As the delivery strategies of peptide drugs using nanocarriers are not comprehensively discussed in many articles, a summary reporting recent approaches was needed. This review consolidates the current understanding of nanosystems in the enhancement of therapeutic peptide efficiency. Here, we point out the main classes of nanoparticles that have been introduced for delivery of therapeutic peptides, and after that, we mention recent advances in each class.

* Correspondence should be addressed to: Prof. Guangjun Nie: niegj@,nanoctr.cn, or Prof. Khosro Khajeh: khajeh@modares.ac.ir

\# These authors contributed equally to this work. 


\section{Summary of relevant literature}

New insights into the therapeutic applications of peptides

Peptide-based therapeutics constituting a unique class of biopharmaceuticals have attracted great interest in the pharmaceutical industry since the advent of insulin therapy in the $1920 \mathrm{~s}^{1}$. Therapeutic peptides have been used for treatments of a wide assortment of diseases, from metabolic and immunologic disorders to cancer and infectious diseases ${ }^{2-4}$. From the late $20^{\text {th }}$ century, over 60 therapeutic peptides have been approved for clinical usage in the United States, Europe, and Japan ${ }^{5}$, and the global sales of therapeutic peptides exceeded USD 70 billion in $2019^{6}$. According to the "Global Peptide Therapeutics Market \& Clinical Trials Insight 2026 " report, there are approximately 800 peptide drugs in clinical trials and 197 peptidebased drugs commercially available on the mar$\operatorname{ket}^{7}$. It is expected that the peptide drug market will keep continuous $9.1 \%$ growth per year until $2026^{8}$

Peptides are arbitrarily defined as biopolymers with 2 to 50 amino acid residues and less than $6 \mathrm{kDa}$ molecular mass 9 . The length of therapeutic peptides is determinative for their secondary structure, cytotoxicity, stability, and mode of action ${ }^{10}$. Most of the previous therapeutic peptides entering the clinical trials stage contain less than 10 amino acids that 7 to 8 of them were used to form amphipathic structures ${ }^{11}$. However, advances in manufacturing technology have provided peptide drugs without length related constraints. Depending on their amino acid sequences, site-specific modifications, and subsequent spatial conformations, peptides retain diverse three-dimensional structures and possess a variety of important physiological roles which are due to their ability to bind with exquisite specificity to the respective biological counterparts. Disrupting the interactions of proteins, propagation or inhibition of signaling cascades, and modulating the plasma membrane integrity are some instances of peptide strategies as therapeutic agents $^{12}$. Furthermore, peptides that mimic natural peptide hormones are considered as a medication by compensating hormone deficiencies in body ${ }^{8}$. The intrinsic property of peptides provides great benefits such as notably lower side effects and less toxicity toward normal tissues compared with chemo drugs or protein therapeutics $^{13}$. However, the number of available side chains, needed for possible peptide conformations and strong target binding, is limited by the short length of polypeptides ${ }^{14}$. Advancements in biotechnological techniques and recombinant DNA engineering lead to the creation of several new artificial bioactive peptides and the establishment of new strategies to conformationally stabilize these peptides ${ }^{15}$. Additionally, this new class of therapeutics is associated with lower production complexity and is more cost-efficient ${ }^{16}$. Through the development and manufacturing processes of peptide production, the quality of final products must be assessed and their lot-to-lot consistency mus be assured ${ }^{17}$. Peptide-related impurities including degraded products and residual solvents, must be precisely controlled during peptide drug production based on the guidelines. The proposed threshold of these impurities usually depends on the type of clinical application (e.g., therapeutic, vaccine, diagnostic) of the peptide ${ }^{18}$.

Despite promising therapeutic properties, naturally occurring peptides are facing several interrelated pharmaceutical limitations, such as short plasma half-life and low oral bioavailability that make them unsuitable for direct use as conventional medicines ${ }^{19}$. In the most frequently employed methods of administration, currently available therapeutic peptides are delivered through the parenteral routes ${ }^{20}$. Lower stability and short circulation half-life of peptides in the bloodstream, in comparison with protein therapeutics, leads to a low drug concentration and consequently, low therapeutic efficiency at the site of action. To overcome this issue, frequent injections are required. On the other hand, multiple injections bring about an oscillating concentration of drug in the blood and also consume higher doses of drugs, resulting in the development of side effects ${ }^{13}$. Potential unwanted immunogenicity is another drawback that should be taken into account. Immunogenicity of peptide drugs that refer to the undesirable immune response in patients has been associated with the systemic (usually parenteral) administration of these therapeutics ${ }^{21}$. Over the past decade, several alternative formulation routes, including oral, respiratory, transdermal, and ocular, have been established and were considered as notably less invasive routes 
for the administration of peptide medicines. Nevertheless, these alternative routes are also limited because of poor membrane permeability, insignificant bioavailability after administration, intrinsic weakness in front of enzymatic digestion of protease, and high clearance rate $^{22}$.

Several techniques have been developed to overcome these drawbacks and increase the stability of peptides in biological fluids. The most commonly used strategy is to add stabilizing agents or a combination of specific antiproteases to the formulation of peptide medicines. These stabilizing additives, including heparin, chelating agents, and hydroxypropyl cyclodextrins, can enhance the thermal stability of peptides ${ }^{23}$ or shield them from aggregation and proteolytic degradation ${ }^{24,25}$. In a complementary approach, conjugation of inert polymers such as polyethylene glycol (PEG), polyvinylpyrrolidone, and albumin to the therapeutic peptides can decrease the immunogenicity of peptides and therefore, confer resistance to neutralization by antibodies ${ }^{26}$. Designing modified peptides that are resistant to enzymatic degradation also shows increased stability in therapeutic peptides ${ }^{27}$. Although these stabilizing methods help to improve the therapeutic index of peptides, in vivo applications are still limited due to their low-throughput pharmacokinetic properties. One approach for increasing the efficacy of peptide drugs without raising the overall dose is to increase the local concentration, particularly by conjugating them with targeted delivery systems such as nanoparticulate vehicles. To date, several types of nanosystems as carriers of proteins and peptides have been developed $^{28}$. Encapsulation of peptides in carrier systems or immobilizing them on the surfaces of nanoparticles have tremendous potential to increase the bioavailability of therapeutic peptides and potentiate their efficacy at the site of action. Here we present an overview of existing nanosystems that have been recently developed for the delivery of therapeutic peptides.

Nanosystems for therapeutic peptide delivery

Incorporation of drugs or other active ingredients into nanocarriers holds enormous promise for further enhancements in the diagnosis and treatment of diseases ${ }^{29}$. Recent advancements in the field of therapeutic peptides have focused on the development of effective peptide delivery to the extracellular or intracellular targets. However, low permeability of peptides through biological barriers and their inefficacious cellular internalization have hampered the clinical translation of peptide-based medicine $\mathrm{s}^{30}$. Therapeutic peptides engineered into nanostructures not only provide opportunities to accelerate smart transportation to the desired site but also prolong their half-life in the plasma. These nanostructures are stable as colloidal particles in aqueous suspensions and are smaller than $1000 \mathrm{~nm}$ in diameter that have been developed for interaction with biological macromolecules and other active substances. Nanoparticles have shown the potential to serve as a conjugate scaffold or provide a cavity for entrapment of biologics to improve their functionality ${ }^{31}$. Efficient cellular internalization, stable spatial architectures, and enhanced vasculature accessibility of nanoparticles hold great potential to overcome the pharmaceutical obstacles of peptides and have motivated the development of peptide-derived therapeutic nanomedicines.

Nanoparticles can be modified to possess stimuli-responsive properties and release their cargo in response to endogenous or exogenous triggers $^{32,33}$. The controlled release of therapeutic peptides from nanoparticles is regarded as an effective strategy for improving their therapeutic index through the reduction in off-target effects. Experimental outcomes complemented by computational simulation demonstrate that physical properties of nanoparticles (e.g., exposed surface area and charge density) are dominant in the interaction of nanoparticles and peptides, and can be engineered to indicate the release profile of peptide drugs ${ }^{34,35}$. Various biocompatible nanoparticle-based carrier systems, mainly polymer-based nanoparticles, lipid-based nanoparticles, inorganic-based nanomaterials, bioactive peptide assemblies, and hybrid nanostructures have been reported for successful targeted peptide delivery (Figure 1). Conjugation of proteins and peptides to these nanoparticles can greatly facilitate their penetration into the target cells and tissues. To improve therapeutic outcomes an effective release of peptide therapeutics from nanosystems must be precisely managed. In the following, we summarize the main functionalities of nanoparticles to improve the therapeutic index of peptide drugs. 


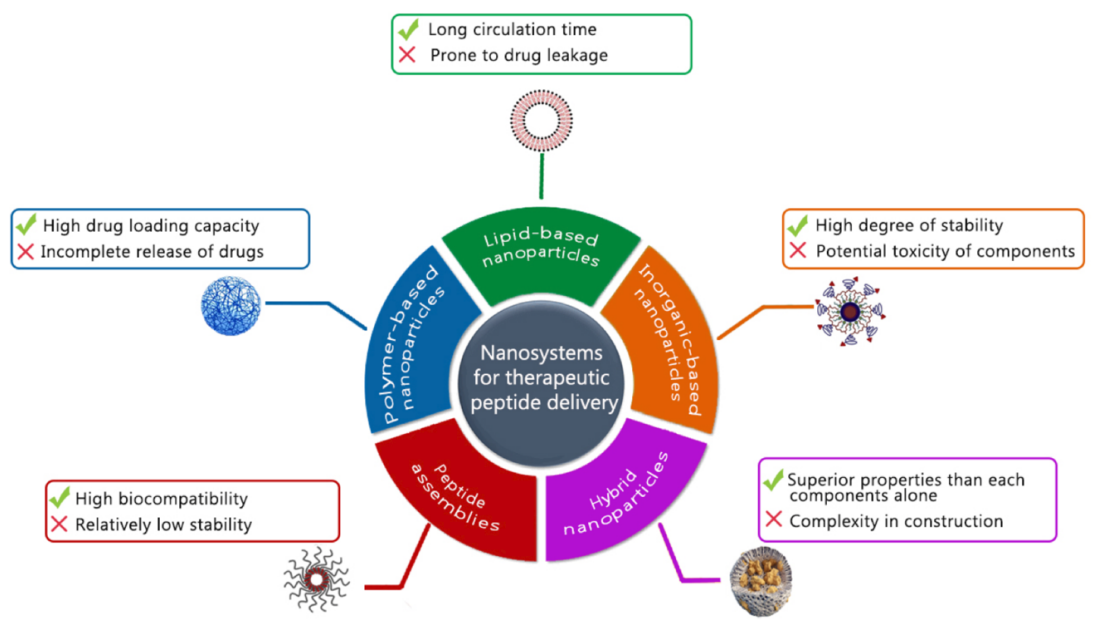

Figure 1: Main classes of nanosystems that contributed to the delivery of therapeutic peptides. As nanotechnology develops, peptide drugs achieve a better pharmacological efficiency via nano delivery systems. Five main classes of nanosystems employed in the delivery of therapeutic peptides are represented here. More expanded definitions and examples are introduced in this review.

\section{Polymer-based nanoparticles}

As a well-established drug delivery system, polymer-based nanoparticles have demonstrated significant advantages over other systems. The term polymeric nanoparticle includes both nanocapsules and nanospheres with size ranging from 10 to $1000 \mathrm{~nm}$ (Figure 2) Nanocapsules consist of colloidal nanobubbles in which the liquid core (oily or aqueous) is enclosed by layers of polymeric membrane that confers reservoir properties to this structure ${ }^{37}$. Increasing the oral bioavailability of peptide drugs using polymeric nanocapsules have been the object of research since $1990^{38}$. Nanospheres are homogenous matrix systems in which the drug is physically and uniformly dispersed throughout the polymeric particle ${ }^{39}$. The amorphous or crystalline nature of nanospheres provides the capability to release the drug in a slow and sustained manner.

Physical properties and drug-loading capacity of different polymeric nanoparticle constructs are significantly affected by the nature of monomers as well as the preparation procedure of polymeric nanoparticles. Synthetic and natural polymers investigated for proteins and peptides delivery mainly includes biodegradable polyesters (e.g., poly [lactide-co-glycolic acid] $[\text { PLGA }]^{40}$, polylactic acid $[\mathrm{PLA}]^{41}$ ), poly (methyl methacrylate), poly (methyl vinyl ether maleic acid) (P[MVEMA $])^{42}$, dextran, hyaluronic acid, and chitosan.

Chitosan (CS) and its derivatives are a wellknown group of natural polysaccharides with high biocompatibility and $\mathrm{pH}-$ responsive feature which have the potential to serve as a carrier for oral delivery of peptides ${ }^{43}$. Moreover, an abundant number of amino and hydroxyl groups on the chitosan backbone provides the ability to capture peptide-based therapeutics via both hydrogen and covalent bonds ${ }^{44,45}$. To date, many ongoing studies have been done to make stable complexes of chitosan and insulin to efficiently enhance their intestinal adsorption and extend their blood duration. ${ }^{46,47}$ 


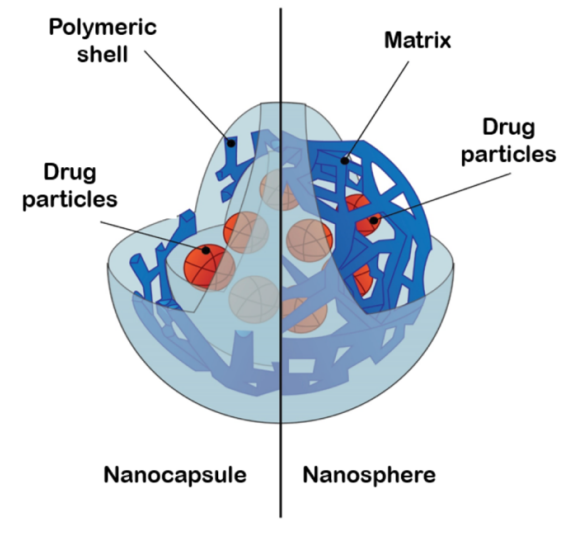

Figure 2: Schematic representations of a polymeric nanocapsule and nanosphere. Nanocapsules consists of a single or multilayered polymeric shell and a liquid core in which payloads are confined to a central cavity of the nanocapsule. However, nanospheres are uniform structures that are formed by dense uniform polymeric matrixes without any empty cavity and drugs uniformly disperse within their matrix.

Accordingly, adequate mucoadhesion through oral administration and self-sustained release profile of insulin loaded chitosan-mucin nanospheres were reported by M.A. Mumuni ${ }^{48}$. Mucin, a natural anionic polymer, rectifies the high degradation rate of chitosan by forming network complexes and therefore reduces the unwanted release of insulin before reaching the adsorption site. High encapsulation efficiency $(90 \%)$ and good loading capacity $(23 \%)$ of these nanoparticles are presumably attributable to the ionic interactions of mucin and chitosan. Other combinations of biopolymers with chitosan to improve its inherent limitations were studied by A. Blachman et al. ${ }^{49}$ They recently introduced an interesting nanosystem that incorporated an egg-derived anti-inflammatory tripeptide (Ile-Arg-Trp) into the matrix of the dermatan sulfate/chitosan nanospheres. Selective targeting toward inflamed endothelium, effectual cell internalization, and intracellular release of peptides were mediated by dermatan sulfate molecules on the surface of nanospheres. Thus, simple and reproducible complexation of peptides with a physically stable structure based on chitosan biopolymer provided a versatile platform for active peptide drug loading.

Hyaluronic acid (HA), a natural glycosaminoglycan polymer, could be a suitable candidate for the construction of polymeric drug delivery systems as a consequence of its biocompatibility and biodegradability. The potential mucoadhesive property, high water-binding capacity, and pseudoplastic behavior of HA have been successfully employed to increase the bioavailability of hydrophobic therapeutic peptides in ocular administration. Clinical applications of peptide drugs through ocular administration have been restricted because of their fast clearance from ocular surface ${ }^{50}$. Cyclosporine A (CsA) is one of these highly hydrophobic peptides which has been recommended to use in the treatment of ocular autoimmune disease $\mathrm{e}^{51}$. Hyaluronic acid-coated poly-3-caprolactone nanospheres loaded by CsA resulted in the increased residence time and sufficient drug concentration in the cornea of the eye tissue and shows promise for local treatment of corneal disease ${ }^{52}$. Alternative polymeric nanoparticles have also been evaluated for entrapment and local delivery of therapeutics. A novel multilayered polymeric nanomicelle formulation composed of polyoxyethylene hydrogenated castor oil 40 (HCO-40) and octoxynol 40 (OC-40) have been designed for ocular delivery of the octreotide as a small therapeutic peptide ${ }^{53}$. In comparison to other similar polymeric structures, using organic solvents instead of conventional aqueous systems in the encapsulation step of this formulation increased the efficiency of peptide encap- 
sulation (up to $76 \%$ ). Generally, successful implementation of polymeric nanoparticles to encapsulate sufficient amounts of cargo has opened a new window in the ocular administration of peptide therapeutics in their native forms.

PLGA based nanoparticles have been widely used for encapsulation and delivery of therapeutic peptides as these polymers are biodegradable and have been approved by the US Food and Drug Administration (FDA) ${ }^{54}$. However, chemical reactions between nucleophilic primary amines in peptide sequences and carboxylic acid end-groups of PLGA or PLGA degradation products form acylated derivatives of peptides, which may cause loss of activity, immunogenicity, and toxicity ${ }^{55}$. It has been demonstrated that peptide PEGylation or co-encapsulation of stabilizer agents including soluble multivalent cationic salts and/or carboxyl methyl chitosan, can strongly prevent the acylation of encapsulated peptides and consequently, enhances the effectiveness of peptide delivery ${ }^{56}$. The effects of a variety of additives such as carbohydrates, amino acids, and surfactants on the stability and encapsulation efficiency of the exenatide-loaded PLGA particulate system was determined by $\mathrm{H}$. Park et al. ${ }^{57}$ They reported that nonionic surfactants, especially poloxamer 188 , can preserve the biological conformations of buried peptides within the polymer by preventing their aggregation at the hydrophobic surfaces and also effectively decrease peptide hydrolysis. A precise combination of additives such as poloxamer 188 , sucrose, and amino acids like phenylalanine provide synergic improvements in the encapsulation efficiency and control the initial burst release of peptide cargos from PLGA particles.

Cell-penetrating properties of guanidine functional groups inspired the design of an effective polyarginine-based nanoparticle for oral delivery of therapeutices ${ }^{58}$. Interaction of polyarginine with tight junction proteins favors the paracellular transport of drugs and improves the transport of therapeutic peptides across the epithelial barriers. Encapsulation of elisidepsin, a cyclic peptide with antitumor activity, within polyarginine nanocapsules, has been explored by G. Lollo et $\mathrm{al}^{59}$. High encapsulation efficiency (close to $50 \%$ ), reasonable stability in intestinal fluids, and enhanced penetration of nanocarriers through the transcellular pathway have made this polymeric nanosystem a potential candidate for use as an oral nanoparticulate delivery system.

The ultimate goal in using polymer-based nanoparticles for sustained delivery of therapeutics is designing a suitable device with an admirable capacity of holding and releasing active agents with the desired rate ${ }^{60}$. Such delivery systems provide concurrent degradation of nanoparticles and release of drugs that extend the active duration of drugs compare to conventional dosage forms. Lately, the effectiveness of polymeric hydrogels in this area has been approved. Polymeric hydrogel systems are a network of cross-linked polymers with the capacity to entrap high concentrations of therapeutic peptides within their hydrophilic cores and prevent peptide degradation over a long period ${ }^{61}$. In a recent study, Exendin-4 (Ex-4), recombinant glucagon-like protein-1 (GLP-1) receptor agonist, was encapsulated in a protamine conjugated poly(organophosphazene) (ProCP) hydrogel system to enhance glucose-dependent insulin secretion in diabetic patients ${ }^{62}$. Ionic interaction of the protamine moiety with Ex-4 has provided a favorable condition for slow drug release and suggested a long-term effective nanomedicine for anti-diabetic treatment. More importantly, this nanocomplex makes concordance in periods of Ex-4 release and ProCP hydrogels biodegradation, then carriers do not remain in the blood longer than the drug release period and injections can be repeated without toxic accumulation of hydrogels during the treatments period (Figure 3).

However, several drawbacks may limit the feasibility of polymeric structures in vivo. The slow rate and incomplete release of drugs from polymeric nanoparticles in line with low rates of biodegradation are the main limitations of polymeric drug delivery platforms. Besides, regarding the high molecular weight nature of polymeric nanoparticles, undegraded polymers accumulate in tissues that could augment inflammatory reactions. Particle size and surface morphology of polymeric nanoparticles can affect their dissociation and biodegradation rates, thereby controlling the efficiency of drug loading and drug release. 


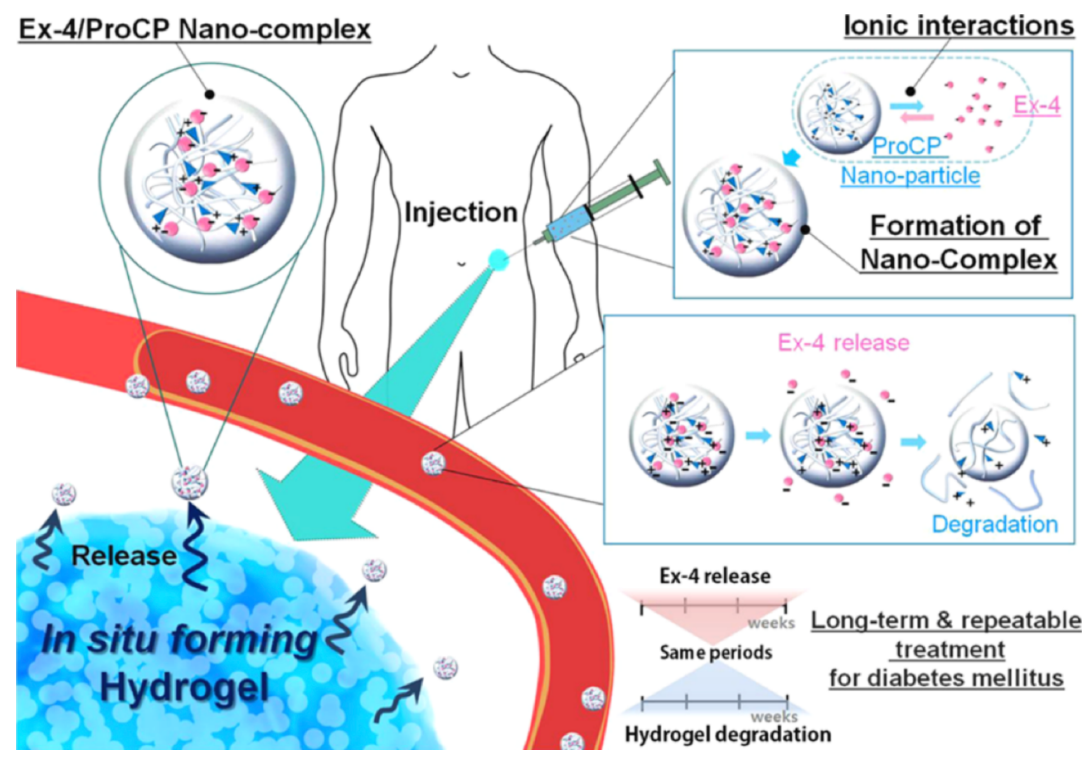

Figure 3: Schematic illustration of protamine-conjugated polymer (ProCP) hydrogels encapsulated Ex-4 recombinant peptide. The formation of a bulk hydrogel in this design is temperature-dependent and happened in situ after injection. Ionic interactions between the hydrogel backbone and Ex-4 drastically enhanced the bioavailability of the drug and provided longterm drug release. Concordant degradation of the Ex-4/ProCP nanocomplex and drug release have the benefits of enabling the nanosystems to be used as repeatable anti-diabetes treatment. Adapted with permission from 62. Copyright (2019) American Chemical Society.

Recent advances in polymer chemistry research are transforming the synthesis methods and application of polymeric nanoparticles that develop several novel formulations with suitable size and surface chemistry to synthase an appropriate carrier for therapeutic peptides. By considering multiple factors at the pre-formulation step to adjust the interaction of peptides and polymer backbones, the kinetics of drug release can be controlled, and the initial burst drug release can be prevented ${ }^{63}$. For instance, co-encapsulation of peptide drugs and relevant stabilizing agents in polymer particulate systems have pivotal roles in the improvement of peptide stability during formulation and delivery $^{64}$. Difficulties in scale-up, complicated steps of modification, and the high cost of synthesis are also significant restraints of polymerbased nanocarriers for their clinical translation. Currently, research efforts have been focused on the optimization of some polymer synthesis techniques such as nanocrystallization ${ }^{65}$ or flow-based methods ${ }^{66}$ which can be easily scaled up to produce large quantities of well- characterized polymeric nanoparticles. Hindered consistency in polymeric nanoparticles that have happened as a result of heterogeneity of polymeric structures also can be addressed by precise control of the synthesis procedures ${ }^{67,68}$. Eventually, the nature of polymers besides toxicological features of them, and careful selection of solvents should be considered to have acceptable final polymeric nanomedicine both by clinicians and patients ${ }^{69}$.

\section{Lipid-based nanoparticles}

Liposomes and other lipid-based colloidal nanoparticles, such as solid lipid nanocarriers, bear the advantage of being in the group of biocompatible nanomaterials, and their specific architecture and properties make them feasible for in vivo applications ${ }^{70}$. Lipid nanoparticles are among the most extensively explored platforms to provide a long-acting system for local peptide delivery for both hydrophilic and hydrophobic drugs (Figure 4) $)^{71}$. Lipid nanocarriers can be modified with targeting moiety to increase their specific ability to pass through the target membrane and could be densely packed 
with a layer of PEG molecules to prolong their blood circulation time via limiting systemic clearance. Several lipid-based formulations for drug delivery have been approved by the FDA, and many products are under different stages of clinical trial ${ }^{72}$.

Liposomal nanomedicines are highly beneficial to improve cellular uptake of peptide drugs once these drugs are loaded into liposomes. These nanoparticles consist of an amphiphilic phospholipid bilayer surrounding an aqueous core cavity resembling the mammalian cell membrane and have higher loading capacity in comparison to other lipid passed nanoparticles. Strong interaction between liposomes and cell membranes guarantees efficient membrane permeability of encapsulated peptide medications $^{73}$. An ultra-flexible liposome to convey a sufficient dose of salmon calcitonin (sCT) through intranasal administration has been formulated by Chen et $\mathrm{al}^{74}$. $\mathrm{sCT}$ is a clinically effective peptide for the regulation of calcium homeostasis. In this formulation, deformation and penetration of liposomes into the nasal mucosa during their residence in the respiratory cavity significantly increased the absorption and bioavailability of the drug compared with $\mathrm{SCT}$ solution. High deformability and flexibility of liposomes in this formulation were correlated to the sodium dodecyl sulfate content in liposomes that increased the absorption of nanoparticles through the pores between the cells of the biological membranes. L-CsA is an example of an aerosolized liposomal formulation that encapsulates cyclosporine A for the treatment of chronic rejection in lung transplant recipients that is currently under phase III of the clinical trials $^{75}$.

Liposome-based chemotherapeutics for cancer treatment possess a large proportion of clinical-stage nanoparticles with high pharmacological potency. One of the most interesting anticancer therapeutic payloads of liposomes are cytotoxic peptides hereupon varieties of their formulations are now under clinical trials. Mepact $^{\circledR}$ (formerly known as L-MTP-PE) is the liposomal muramyl tripeptide formulation that has been approved for parenteral administration in patients with osteosarcoma ${ }^{76,77}$. Preclinical studies confirmed that this formulation has at least 10 times less toxicity than free drug substances. Combinational therapy of L-MTP-PE with conventional chemotherapeutics also provided benefits for the treatment of osteosarcoma $^{78}$. Studies on Stimuvax ${ }^{\circledR}$ (earlier known as BLP25), which was another example of anticancer liposomes incorporating an antigenic lipopeptide (tecemotide), has been terminated in phase III of the clinical trial, as it failed to improve overall survival in non-small cell lung carcinoma patients ${ }^{79}$. Modification of Stimuvax ${ }^{\circledR}$ by incorporating a synthetic immunoadjuvant peptide is under more investigations to come into clinical studies ${ }^{80}$.

The precise liposome peptide formulation and the peptide behavior in the liposome are critical to control the peptide release rate. The formulated nanoparticles developed by N. Rezaie et al. were a liposome-based anti-angiogenesis peptide delivery system to enhance the stability and plasma half-life of the endostatin peptide ${ }^{81}$. It has been demonstrated that changing the molar ratio of phospholipid and peptide payload (endostatin) can affect the release profile of the encapsulated drug by changing the physicochemical properties of the liposomal system. Based on their results, an increase in surface area of liposome and, therefore, higher interaction between peptide payload and lipid bilayer were the consequences of the smaller size of liposomes, which increased the release rate of the peptide cargo. On the other hand, self-assembly of peptides in the hydrophilic core of liposome, which has been simulated through molecular dynamics, is an effective factor in prolonging the peptide release rate in vivo ${ }^{81}$. A few great examples of liposomal formulations for peptide-based drug delivery have been summarized in Figure 4.

Lipid nanoemulsions are non-covalent lipidbased aggregates with low antigenicity and potent applications in the ocular drug delivery ${ }^{87}$. Bioadhesive properties of cationic lipid-based nanoemulsions cause stable electrostatic interactions with the cells of the ocular surface and lead to the successful development of the novasorb $^{\circledR}$ technology, which has effectively treated ophthalmic diseases with controlled drug release manner ${ }^{83}$. The advantages, such as easy fabrication and small size for transcorneal penetration, are combined with mucoadhesive features and introduced an ideal nanoemulsionbased ocular delivery ${ }^{88}$

Solid lipid nanocarriers are classified as colloidal particles with lipid core matrix consisted 
of solid lipid nanoparticles (SLNs) and nanostructured lipid carriers that have a higher loading efficiency for hydrophobic drugs compared with liposomes ${ }^{89}$. Among the most recent formulations, SLNs have been introduced as a well-organized and non-toxic alternative lipid nanoparticle carrier system that can be conveniently dispersed in water or aqueous surfactant solutions. SLNs can improve the blood-brain barrier permeability of loaded drugs and execute a sustained release of drug payloads ${ }^{90}$.

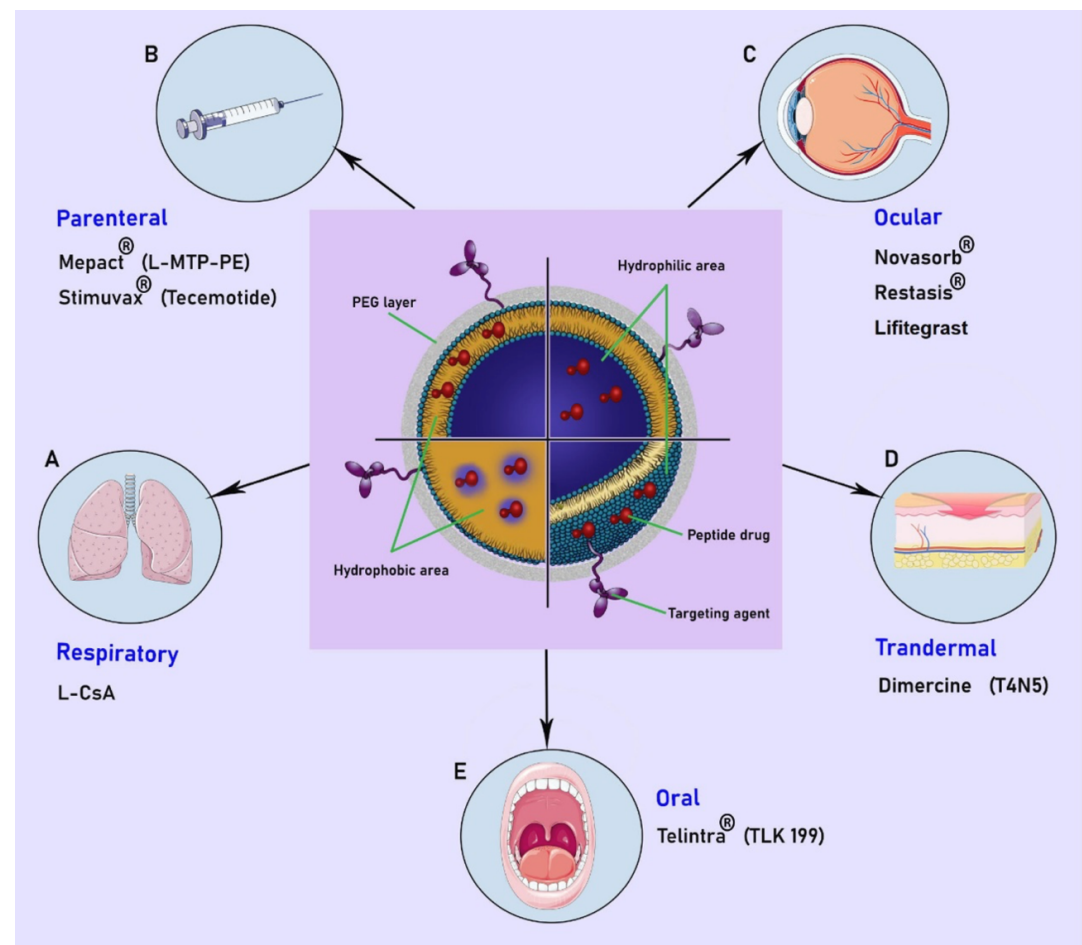

Figure 4: Lipid-based nanoparticles are developed for therapeutic peptide delivery through diverse administration routes. (Center) Multifunctional lipid structures commonly are coated with a layer of PEG and are decorated by targeting agents for long plasma circulation and specific tissue accumulation. Lipid-based nanoparticles provide separate areas for encapsulation of both hydrophilic and hydrophobic drugs. (A) Inhalation delivery of CsA through liposomal formulation (L-CsA) was intro-

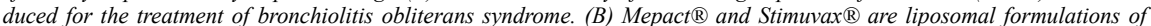
peptides with anticancer activity. (C) Nanomicellar solution of lifitegrast peptide 82 and nanoemulsion formulations of CsA peptides with anticancer activity. (C) Nanomicellar solution of lifitegrast peptide $e^{82}$ and nanoemulsion formulations of CsA
(Novasorb ${ }^{\circledR}$ and Restasis $\left.{ }^{\circledR 8}\right)^{83,84}$ are novel formulations for the treatment of dry eye diseases which are developed for ocular administration. (D) T4N5 (T4 endonuclease V) liposomal lotion (also known as dimercine) has been approved for lowering the risk of skin cancer in patients with xeroderma pigmentosum through the transdermal administration85. (E) Oral administration of ezatiostate through the liposomal formulation is known as Telintra ${ }^{\circledR}$ (TLK199) that is under the development for the treatment of myelodysplastic syndromes ${ }^{86}$.

Antibacterial peptides are one of the most common peptide payloads of clinically relevant lipid-based nanoparticles. Co-encapsulation of vancomycin and potent antioxidant ellagic acid within SLNs were found to significantly reduce the nephrotoxicity of this antibacterial glyco- peptide $^{91}$. Physical stability and drug-lipid compatibility of this combination provided a slow release of active antibiotics with minimum nephrotoxic effects. Surface modification approaches such as PEG coating and ligand binding can be adapted directly to SLN formulations to respectively increase their bioavailability and 
specificity toward the site of interests ${ }^{92}$. Furthermore, decorating the surface of nanoparticles by functional peptides confers exclusive properties to the SLN based delivery platform. To emphasize the importance of endosomal escape for orally delivered peptides, Y. Xu et al. introduced an SLN with the incorporation of hemagglutinin-2 (HA2) on the solid lipid shell for oral delivery of insulin ${ }^{93}$. HA2 is a non-toxic fusogenic agent that confers the ability of endosomal membrane destabilization to the nanoparticle and facilitates the transepithelial transport of the nanocarrier ${ }^{94}$. In vitro and in vivo studies have demonstrated that administration of these SLN loaded HA2 with loading capacity close to $7 \%$ is enough to maintain the highest biological activity of internalized insulin. In another study, decorating the surface of SLNs with the L or D stereoisomers of cell-penetrating peptides represented increased adsorption of insulin through the intestinal mucosa ${ }^{95}$.

Lack of robust control of drug release, limited drug-loading capacity, and not enough stability after administration are the main disadvantages of lipid-based nanoformulations ${ }^{96}$. Manipulation of interior $\mathrm{pH}$ of liposome through co-encapsulation of stabilizing agents can result in excellent retention of therapeutics inside the core cavity of liposomes. Bile salt stabilized liposomal carrier, bilosomes, are the widely accepted strategy to improve the stability and permeation of peptide drugs in gastrointestinal (GI) tract ${ }^{97}$. Furthermore, covering the outer surface of lipid nanoparticles with a layer of polymers enhances the stability and helps in steric stabilization ${ }^{98}$.

The use of toxic organic solvents in the synthesis of lipid nanoparticles was another limitation in the clinical use of these nanomedicines that has recently been addressed through using alternative less toxic supercritical fluids. The low solubility of peptide drugs in the lipid matrix also limits the incorporation of peptidebased medicines ${ }^{99}$. Current studies on lipidbased nanoparticles not only focus on liposomes or solid lipid nanocarriers, but there are many studies that are developing advanced approaches for using lipid nanoparticles as therapeutic peptide carriers. Accordingly, high potential of ultra-flexible particles such as transfersomes and invasomes for transdermal penetration has been gaining considerable attention in the treatment of different skin cancers ${ }^{100,101}$.
Transferosomes and invasomes have the same structural constituent as liposomes and consist of phospholipids, ethanol, and hydrocarbon compounds that confer deformability to the structure $^{102}$. Expanding an appropriate technology for the industrial production of these novel particles is required for future clinical application of them.

Inorganic-based nanoparticles

The structural and physical properties of inorganic-based nanoparticles can easily be tailored to exhibit distinct interaction with biological systems. Inorganic NPs can provide a multifunctional platform by incorporation of therapeutic peptide and targeting ligand simultaneously ${ }^{103}$. Most inorganic nanoparticles have several unique characteristics, such as tunable optical properties, large surface area, and high stability compared with organic material that can add valuable functionality to the nanomedicines $^{104}$. Conjugation of peptides to inorganic nanoparticles particularly has been widely used to provide higher physical stability of these nanoparticles ${ }^{105}$. Therapeutic peptides can be attached on the surfaces of inorganic nanoparticles with a covalent bond or a non-covalent interaction, increasing their stability in relation to proteolytic degradation. Additionally, self-assembly of inorganic nanoparticles has resulted in stable higher-ordered structures that can entrap therapeutics in the core cavity of the nanoassemblies ${ }^{106}$. Hence, inorganic nanomaterials, including silica-based nanomaterials, metallic nanoparticles, quantum dots (QDs), and carbon-based nanomaterials provide unprecedented prospects in biotechnology and nanomedicines. Polar characteristics of nanomaterials, including inorganic nanoparticles, prevent their diffusion through the cell membrane, therefore cellular uptake of these materials proceeds mainly via endocytosis ${ }^{107}$ (Figure 5). During the endocytosis process, nanoparticles are captured into the vesicular compartments (i.e., endosomes) followed by endosomal scape to deliver peptides or other therapeutics to the cytosolic compartments. Mesoporous silica nanoparticles (MSNs) with large surface area and porous interior have provided high loading capacity for entrapment and delivery of bioactive materials and peptides ${ }^{108}$. Electrostatic or hydrophobic interactions of proteins and peptides with the hollow cavity of MSNs provide peptide-based drugs loading with an intact three- 
dimensional structure and be protected from degradation by proteases. Textural properties of MSNs (surface area, pore-volume, and surface charge) are major factors that contribute to the loading of therapeutic peptide and determine their membranes interactions ${ }^{109}$. Strong electro- static adsorption of cationic antibacterial peptide inside the pore systems of anionic silica nanoparticles allows for easy formulation and also drastically enhances cellular internalization of the therapeutic peptide ${ }^{110}$.

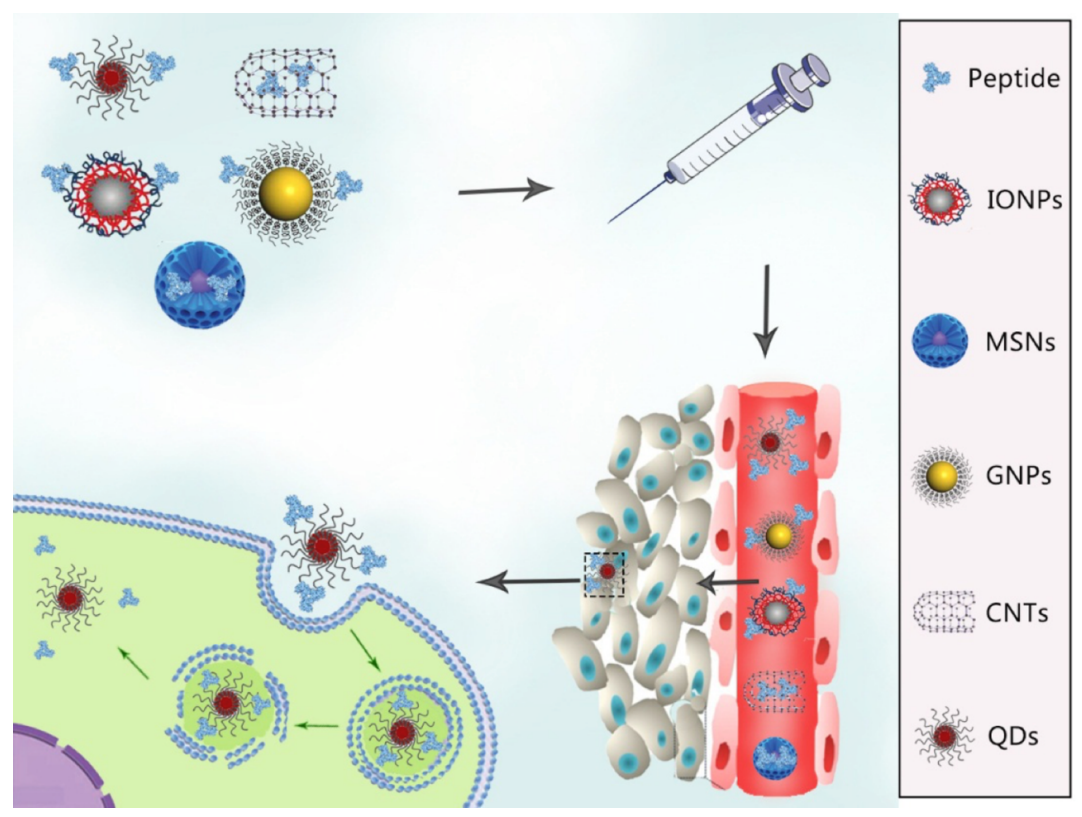

Figure 5: Schematic illustration of widely used classes of inorganic nanoparticles and their application as peptide-carrying vehicles. Inorganic nanoparticles owning their reasonable structural stability and also the possibility for their modification with the array of functional groups are promising candidates for conjugation with therapeutic peptides since they prolong their bioavailability in the circulation after systemic administration and mediate their intracellular delivery. IONPs: iron oxide nanoparticles; MSNs: mesoporous silica nanoparticles; GNPs: gold nanoparticles; CNTs: carbon nanotubes; QDs: quantum dots.

Modification of a porous silica matrix with hydrophobic or stimuli-responsive functional groups enables stable storage and tunable release of the cargo ${ }^{111}$. Hydrophobic characteristic of SH-modified mesoporous silica pore walls improved their interactions with the antimicrobial LL-37 peptide (a cathelicidin-derived peptide with anti-endotoxic activity) and effectively decreased the release kinetic of peptides $^{112}$. The controlled release of antimicrobial agents represents a potent approach to hinder implant-related infections. Gold nanoparticles (GNPs) are at the forefront of the rapidly developing field of biomaterials. Biocompatibility,

ease of surface biofunctionalization, size-dependent optical properties, and longer plasma half-life than peptides in general, offers possible visions for designing conjugated constructs of gold nanoparticles and therapeutic peptides ${ }^{113}$. Peptide sequences containing thiol or amine moieties can be easily conjugated to the surface of GNPs and facilitate multi- functionalization of these nanoparticles with a set of different peptides for simultaneous targeting, diagnostic, and therapeutic functions. This strategy significantly amplifies the specificity of nanosystems and enhances the bioactivity of peptide drugs over the free peptides ${ }^{114}$. A $20 \mathrm{~nm}$ GNP decorated with both targeting peptides, 
and an analogous of the RAF peptide ligand (an inhibitor of tumor growth and angiogenesis) was evaluated by L. Hosta-Rigau et al. for their selectivity toward cancer cells, higher cell internalization properties and improved efficacy of RAF antitumor activity ${ }^{115}$. The same approach also has been employed by A. Kumar et al. for multi-functionalized small ( $2 \mathrm{~nm})$ GNPs with both a therapeutic peptide ( $\mathrm{p} 12$, with loading efficiency close to $65 \%$ ) and a targeting peptide that introduced a monolith system with selective cancer cells binding as well as therapeutic effects ${ }^{116}$. Depending on the peptides structure, conjugation of GNPs with only one type of biomolecules such as peptides (homo-functionalization) may lead to the formation of aggregates with complicated structures and divergent physicochemical properties. To address this issue, an alternative methodology for peptide conjugation was introduced by C.T. Matea et al. In their work, the conjugation of two or more functional biomolecules (hetero-functionalization) prevents undesirable aggregation of peptide coated GNPs ${ }^{117}$. JAG-1 peptide fragment, an agonist in the activation of the Notch signaling pathway, coupled with GNPs surface via direct conjugation as a homo-functionalized system and also conjugated through PEG linker as hetero-functionalized system (Figure 6).

Route I
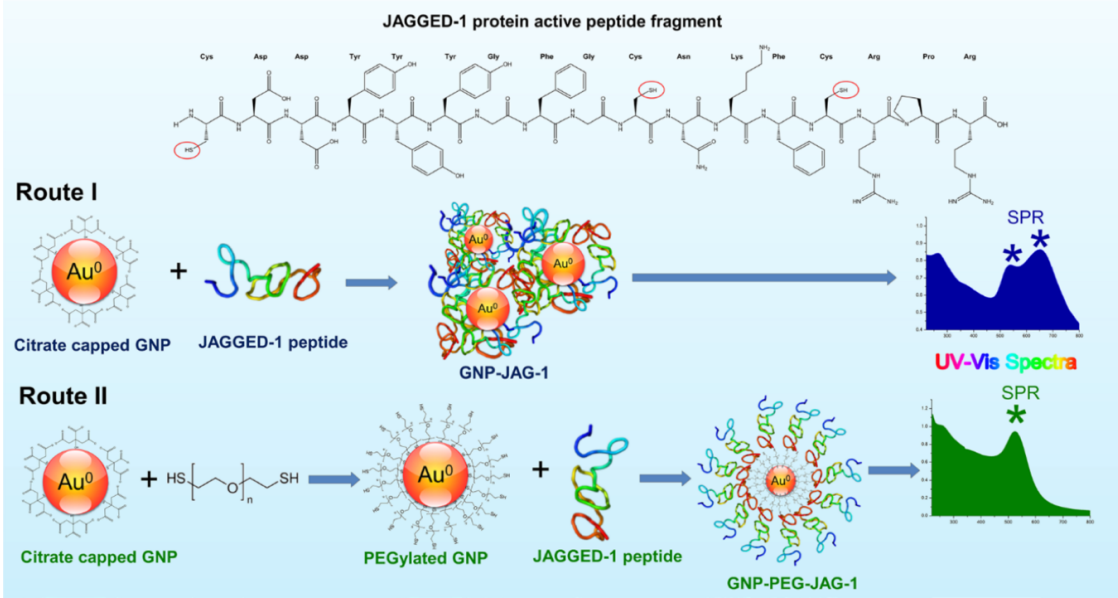

Figure 6: Illustration of the proposed functionalization routes for binding the JAG-1 peptide (top chemical structure) to gold nanoparticles (GNP). In this study, the homo-functionalization of GNPs simply with peptides (route I) has created aggregates of peptide coated GNPs with altered physicochemical properties and high polydispersity index. However, hetero-functionalization of GNPs with peptides and through the PEG molecules spacers (route II) leads to monodisperse stable peptide coated nanoparticles, which would promote an efficient interaction between the JAG-1 peptide and the Notch receptor. Adapted with permission from 117

Comparing the resulting nanoparticles from each route indicated that the direct coupling of peptides on the surfaces of GNPs might not provide monodispersed and well-directed functionalized nanoparticles. While PEGylation of the surface prior to the coupling step makes a spacer between the JAG-1 peptide and the gold core and yields a monodisperse and aqueous stable nanometric structure
It is stipulated that insulin coated GNPs can retain the physicochemical effects of insulin after intravenous administration ${ }^{18,119}$. Using glucan coated biocompatible GNPs for expansion of oral insulin delivery proceeded to phase II clinical trials for type 1 diabetes mellitus by Midasol Therapeutic ${ }^{\text {TM }}$ 120,121.

Semiconductor QDs have emerged as nanocarriers with outstanding optical and chemical properties. They have been widely investigated owing to their excellent potential to 
be used as a probe for diagnosis or as a carrier for traceable targeted delivery of drugs ${ }^{122}$. Therapeutic application of these nanoparticles was demonstrated as self-assembly of fluorinated QDs based on non-covalent fluorine-fluorine interactions which have been used in designing a delivery platform for therapeutic proteins and peptides in their active forms ${ }^{123}$. Effective release of encapsulated therapeutics only in response to the acidic $\mathrm{pH}$ can prevent the drug release outside the cells. On the other hand, QDbased theranostics cater to both therapeutic and diagnostic modalities. Conjugation of two potent antimicrobial peptides with ultra-small tungsten disulfide QDs not only boosts the antimicrobial and anti-biofilm potency of these peptides but also represents a promising tool for selective pathogen imaging ${ }^{124}$. The intrinsic toxicity of constituent ions has restricted clinical utilization of QDs as contrast agents or as nanoscale carriers of therapeutics. Recently emerging non-toxic cadmium-free QDs (e.g., silicon QDs, QDs of group III-V and group I-III-VI, or graphene and carbon QDs) offer safer theranostic formulations which can be used for bioimaging and therapeutics delivery purposes $^{125}$.

Carbon nanotubes (CNTs) are rolled-up graphene-based nanoparticles with great potential for biomaterial conjugation. However, low water solubility and toxicity have limited their biopharmaceutical applications that given rise to the development of CNTs transformation strategies to achieve a viable delivery system. Functionalization of CNTs covalently or electrostatically with proteins and peptides not only can control limiting factors it can also enhance the effectiveness of therapeutic peptides ${ }^{126}$.

Single-wall CNTs (SWNTs) are considered as an ideal carrier system for synthetic peptide vaccines to improve immune responses against human tumor antigens ${ }^{127}$. Peptide-SWNT constructs are non-toxic and were rapidly internalized by antigen-presenting cells which generated effective antibody responses against even weak immunogenic tumor antigens. In one recent study, CNTs conjugated with indolicidin ${ }^{126}$ and other alternative antimicrobial peptides (such as TP359, TP226, and TP557) ${ }^{128}$ resulted in a significantly better antibacterial activity than free peptides. Surface modification of metal nanoparticles with therapeutic peptides can solve some limitations in using these nanoparticles, and it can also increase the stability and effectiveness of the peptides by increasing their plasma half-life.

Inhibition of Clostridium difficile (C. difficile) spore germination is clinically important as it is associated with healthcare-facility-related infections, and after its development, there are only a few antibiotics available to control it ${ }^{129}$. Vancomycin, a glycopeptide antibiotic, is the traditional treatment of choice in this case, but the off-target side effects can contribute to dysbiosis and intestinal inflammation. Singlecrystal non-stoichiometric $\mathrm{Fe}_{3}-{ }_{\delta} \mathrm{O}_{4}$ iron oxide magnetite nanoparticles (IONPs) show unique sporicidal activity and possess a strong binding affinity to the $C$. difficile spores through a hydrophobic interaction ${ }^{130}$. Surface modification of each iron oxide $\mathrm{Fe}_{3}-{ }_{\delta} \mathrm{O}_{4}$ nanoparticles with $5.8 \pm 1.5$ molecules of vancomycin resulted in the spore-targeting van-IONPs formulation with effective suppression of spore germination. Accumulation of the vancomycin-targeted delivery system on the spore surface increases the local concentration of antibacterial peptide and optimizes its efficacy without raising the overall dose.

Aggregation and unfavorable interaction in aqueous solvents are the inherent disadvantages of inorganic nanoparticles that must be addressed prior to the development of inorganicbased nanocarriers ${ }^{131}$. Surface engineering strategies, including coating by small ligands, polymers, and lipids, are long-lasting methodology to enhance the colloidal stability of inorganic nanoparticles in the aqueous solutions ${ }^{132}$. Burying surfaces of the metal complexes or inorganic nanoparticles with protein containers is another appealing approach that has been explained as shielding nanoparticle surfaces by a layer of assembled protein chains to increase their stability and circulation lifetime ${ }^{133,134}$. Further advancements in this technique have been achieved through decorating the surfaces of inorganic nanoparticles by specific cargo-loading peptides ${ }^{135}$. Via lock-and-key interactions, cargo-loading peptides efficiently bind to the inner container surfaces and retain the morphology and integrity of protein during the delivery. Accordingly, protein containers are versatile compartments demonstrating a new direction for the development of hybrid protein-inorganic 
nanoplatforms with higher drug delivery effectiveness. Long-term accumulation in organs of the reticuloendothelial system and non-negligible toxicity are other drawbacks in the application of these nanoparticles that should be considered while developing the inorganic nanomaterials for clinical use. A new generation of inorganic nanoparticles with alternative elemental compositions are currently being developed that possess faster degradation and excretion from the body and, therefore, less toxicity as peptide delivery platforms ${ }^{136,137}$. Nonetheless, the precise equilibrium between the clearance rate and the effective concentration of inorganic nanoparticles in the target tissues is challenging and needs to be investigated in future studies.

\section{Bioactive peptide assemblies}

Self-assembly of peptide and peptide conjugates has been widely reported as highly tailored materials optimized for therapeutic delivery applications ${ }^{138}$. Depending on the biochemical environment, peptides undergo hierarchical assembly through non-covalent interactions and form ordered aggregates, which can be used as a nanocarrier for drug delivery. Furthermore, the peptide itself may have therapeutic properties, both through presented bioactivity and by a drug-like function which interfaces with a specific pathway in cells or tissues ${ }^{139}$. The main advantage of peptide assemblies is their higher stability in circulation, which can significantly enhance the therapeutic index and improve their clinical translation.

A broad class of therapeutic peptide assemblies are peptides amphiphiles (PA) wherein a peptide is grafted with linear or branched nonpeptide modifiers via a linker or spacer to drive self-assembly through hydrophobic collapse ${ }^{140}$. PAs have been widely developed as nanomedicines due to their modularity, biocompatibility, and straightforward synthesis procedures with controlled size and shape. Spherical micellar PA derived nanoparticles have been effectively utilized as nanocarriers for stimuli-responsive delivery of chemotherapeutics ${ }^{141,142}$. However, the intelligent design of PAs for having stimuli responsiveness creates smart delivery platforms for therapeutic peptides. Y. Ding et al. have shown that conjugation of an angiogenesis inhibitory peptide $(\mathrm{C} 16 \mathrm{Y})$ with the hydrophobic molecule, 3-diethylaminopropyl isothiocyanate
(DEAP), creates amphiphilic building blocks with the capability of self-assembling to the stable antitumor nanostructures ${ }^{143} \cdot \mathrm{pH}$-responsive assembly/disassembly behavior of this structure provided intact peptide functionality in the circulation, whereas dissociation of the nanostructure in the tumor site makes peptide sequences well available for binding to its receptor and inhibiting angiogenesis. Considerable serum stability of DEAP-C16Y nanostructures compared with $\mathrm{C} 16 \mathrm{Y}$ demonstrated the efficiency of the self-assembling process for the protection of therapeutic peptides. PA nanocarrier platforms also incorporated in atherosclerosis nanomedicine for targeted delivery of an immunotherapeutic (Ac2-26 ) to atherosclerotic plaque $^{144}$. Ac2-26 is the mimetic peptide of glucocorticoid annexin A1 protein which has protective effects against inflammation within atherosclerotic lesions. Upon exposure to biochemical cues overexpressed in atheroma microenvironment, active Ac2-26 is released and reduces plaque burden of atherosclerotic niche. A related strategy also has been applied by L. Zhang et al. for enhancing the bioavailability of hydrophobic T4 therapeutic peptide (Figure $7)^{145}$. Dual responsive peptide-assembling nanostructures (P-T4) obtained by conjugation of $\mathrm{mPEG}$ modified peptide and angiopoietin receptor targeting peptide (T4) indeed prolonged half-life time of the T4 in the circulation and responded to a decreased level of $\mathrm{pH}$ and cleavage by the endogenous legumain protease at the tumor microenvironment. P-T4 nanoparticles specifically target tumor tissues and block the reconstruction of blood vessels in the tumor. Taking advantage of the higher expressions of specific proteinases such as matrix metalloproteinase-2 (MMP-2) inside the tumor microenvironments, K. Cheng et.al. designed a PA derived nanoformulation that holds the peptide sequence substrate of MMP-2 conjugated to the short D-peptide antagonist of PDL-1( ${ }^{\mathrm{D} P P A}$ $1)^{146}$. Precise and controlled release of ${ }^{\mathrm{D} P P A}-1$ in the extracellular matrix of cancer cells increased the chance of interaction between this peptide and its counterpart, PDL-1, which caused modulation of the immunosuppressive tumor microenvironment and enhanced cancer therapy.

Designing an appropriate self-assembling therapeutic peptide often needs peptide se- 
quence modifications or conjugations. However, these additional modifications may inevitably affect the intrinsic effectiveness of peptide drugs. The rational design of some intelligent nanosystems with the possibility of losing their extra modifications in the site of action can significantly improve their therapeutic outputs. Strong cytotoxicity or inherent hemolytic activity of some anticancer peptides also limits the application of peptide-based nanostructures obtained from these peptides ${ }^{147}$. Making hybrid nanostructures by incorporation of more stable and less immunogenic materials to the bioactive peptide assemblies provide the opportunity of expanding the utilization of these nanoparticles in medicine. a)

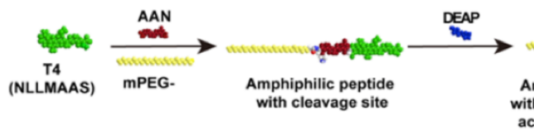

b)

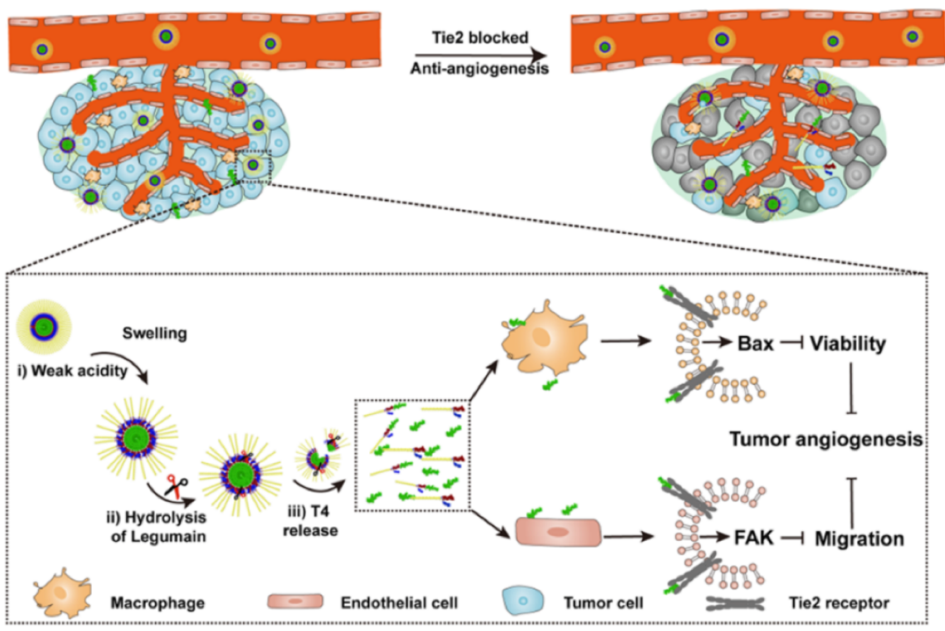

Figure 7: Illustration of fabrication and proposed mechanism of therapeutic peptide-assembling nanoparticles function. (a) Peptide sequences have been synthesized through solid-phase techniques. Self-assembly of peptides in aqueous solution, pH 7.4 is driven by an amphiphilic character of monomer units. (b) Dual responsiveness of nanoparticle to decreased pH and tumor-specific protease kept the nanoparticles intact in blood circulation and caused the accumulation of an active peptide at tumor sites. Adapted with permission from 145. Copyright (2019) American Chemical Society.

\section{Hybrid nanoparticles}

Hybrid nanoparticles are considered as a versatile and continually growing field across a number of biomedical disciplines. Connecting two or more materials at the nanometer scale generates a new hybrid particle that combines the intrinsic characteristics of its components and gains some extra properties caused by synergistic effects between the constituents ${ }^{148}$. Meanwhile, the critical challenge in designing hybrid nanomaterials is keeping or enhancing useful characteristics of the components (e.g., specificity and stability) and reducing their particular limitation (e.g., toxicity and fast drug release).

Polymer coating of liposomes is one of the promising approaches for stabilizing these nanoparticles against unfavorable interactions of liposomal membranes and their fusions. Besides a protective PEG coating ${ }^{149}$, Eudragit E$100^{\circledR}$ (a non-toxic cationic FDA approved polymer) has been applied for coating liposomes with encapsulated antimicrobial peptide 
(Alyteserin-1c) $^{150}$. Enhancement of antibacterial activity of peptide after encapsulation in liposome and coating with polymer suggested a hopeful strategy for designing viable therapeutic options. The combination of natural biocompatible polysaccharides such as chitosan, chondroitin sulfate ${ }^{151}$, and pectin ${ }^{152}$ with liposomes have also previously reported that present reduced undesirable aggregation as well as slower peptide release during storage.

Organic-inorganic hybrid drug delivery systems, usually known as double-layered structures, consist of inorganic core moiety, which provides rigidity or enhanced thermal stability to the soft organic shell ${ }^{153,154}$. Functional groups of the organic moiety (e.g., lipids, polymers, and peptides) may be used to adjust the hydrophobicity and bioreactivity of the inorganic matrixes and prolong their plasma retention time. Y. Jin et al., developed an organic-inorganic nanocomposite with dual-functionality possessing gadolinium-doped MSNs as a core structure coated onto the liposomes ${ }^{155}$. MSNs were further loaded by pro-apoptotic KLA peptides with encapsulation efficiency close to $87 \%$ for efficient intracellular delivery of the cancer theranostic system. Resulting nanocomposites exhibited enhanced colloidal stability, improved biocompatibility, and more effective cellular uptake of the liposome. Engineering inorganic nanoparticles with a polymer surface coating has proved to be an excellent tactic to conquer their disadvantages in vivo. Highlybranched G5 polyamidoamine (PAMAM) dendrimers were applied to cover the large pore sized-MSNs that strongly enhanced specific cellular accumulation and cell-penetration capabilities of inorganic MSNs ${ }^{156}$. Polymeric shells in this designed hybrid platform are employed as gatekeepers and provide cavities for drug loading. Dual-entrapment of anticancer NuBCP-9 peptide on the silica pores and doxorubicin on the PAMAM polymer cavities displayed higher loading efficiency for peptide $(10.8 \%)$ than doxorubicin $(2.1 \%)$ and showed superior antitumor activity in comparison with the monotherapy. Natural biopolymers possess favorable interactions with biological compartments while coating inorganic core nanoparticles and therefore enhance the bioavailability of hybrid nanosystems after administration. Capping insulin loaded GNPs by chitosan ${ }^{157}$, dex- $\operatorname{tran}^{158}$, and chondroitin sulfate ${ }^{159}$ intensifies adsorption of the inorganic core through noninvasive administration routes and reduced the frequency of insulin injection by facilitating the lasting of insulin activity.

Self-assembling nanostructures consisting of peptides and polymers are immensely attractive for the fabrication of multifunctional materials. Potential stimuli-responsive behavior and diverse bioactivity of each component alone are great advantages of these nanocomposites ${ }^{160}$. Stimulus-driven assembly and disassembly of polymer-peptide hybrid structures have been used to build dynamic carriers applicable in tumor treatments ${ }^{161}$. Y. Cong et al. introduced a hybrid polymer-peptide formulation composed of poly ( $\beta$-thioester) backbone that was functionalized by the joined sequences of cytotoxic peptide and $\mathrm{pH}$-sensitive moiety ${ }^{162}$. Hydrophilic property of this hybrid construct owing to the $\mathrm{pH}$-sensitive tail provided deep penetration into solid tumors as a single-chain form. Once reaching the tumor microenvironment, mildly acidic $\mathrm{pH}$ triggers the hydrolysis of hydrophilic tail sequences that leads to the formation of 100 $\mathrm{nm}$ particles through self-assembly of peptide sequences. The transformation from disassembled chains to the assembled structures contributes to the high internalization efficiency, improving the therapeutic activity of the cytotoxic peptide. In another rational design, taking advantage of shape conversion properties of polymer-peptide hybrid structures, HA biopolymers, and $\mathrm{pH}$-sensitive peptide sequences were combined to exhibit successive morphology and size transformations in response to the acidic tumor microenvironment and NIR laser irradiation (Figure 8$)^{163}$. Melittin, a bee venomderived cytolytic peptide with effective anticancer properties, was self-assembled by incorporating a NIR-absorbing photothermal molecule (cypate) and were further coated by HA polymers to form tumor-targeted nanospheres. The resultant hybrid structures were suitable for long-term systemic circulation and also had the potential to be transformed into the nanofibers in response to the acidic microenvironment of tumor cells. Deep penetration into the tumor and durable retention for complete tumor ablation have been achieved as a result of this shape transformation, which also significantly inhibited the metastatic dissemination of tumor cells. 


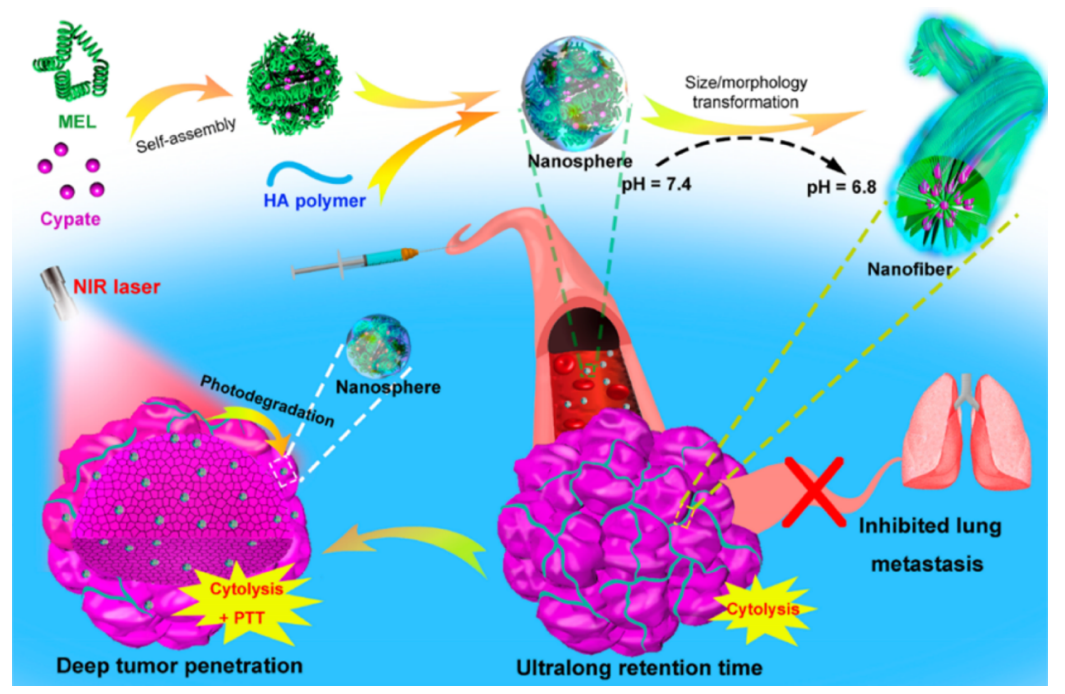

Figure 8. Schematic representation of the fabrication and function of stimuli-responsive hybrid melittin peptide-polymer complex. Self-assembly of therapeutic peptide and further coating by HA biopolymers provided stable nanospheres in circulation with higher accumulation into the tumor tissues. Morphology transformation from the nanosphere to nanofiber happened in response to the acidic $\mathrm{pH}$ of the tumor and caused the ability of deep tumor penetration for more efficient tumor toxicity. Adapted with permission from 163. Copyright (2019) American Chemical Society.

Manufacture of hybrid nanocomposites is limited to small scale productions as designing, modification, and functionalization of these nanosystems can be complicated. Irrespective of the preparation, expanding hybrid particulate systems that can be degraded without producing toxicity and balance the safe and sustainable release of therapeutic peptides is also challenging. Adopting endogenous building blocks in the fabrication of hybrid nanoplatforms can endow adequate biocompatibility to the system and optimize the performance of hybrid nanoparticles according to pathological mechanisms ${ }^{164}$. It has also been proven that the integrity of organic shells of hybrid nanoparticles changes dramatically following their localization in endosomal and lysosomal compartments ${ }^{165}$. The interaction of therapeutic peptide and its localization in the nanosystems should be engineered to control the bioavailability and release rate of therapeutic peptides and to conquer the remaining challenges. Evidently, current reported hybrid nanoparticles are far from being applied clinically; however, extensive efforts are being devoted to tackling obstacles.

\section{Administration routes of peptide nanocarriers}

One of the most important factors in designing the nanomedicines is the modality of administration. The route of administration has a significant influence on the therapeutic outcome of peptide drugs. Nanoformulation of the therapeutic peptides have demonstrated enhanced prospect for systemic delivery. Extensive research both in industry and laboratory are ongoing to develop alternative noninvasive routes to administrate nanoformulations of therapeutic peptides. When considering the route of administration for peptide drugs, matching the delivery route to the therapeutic target location is paramount. Currently, most peptide nanocarriers are administrated by the parenteral injection, albeit it is not the preferred one due to several disadvantages such as low patient compliance. Hence, noninvasive administration forms for delivery are being explored including oral, ocular, respiratory, and transdermal routes that offer alternative cost-effectiveness pathways with higher patient admission. A brief overview of different administration routes of nanocarriers are pointed out below to provide a deeper 
understanding of the biological barriers and the challenges associated with each drug delivery route. Besides, some achievements in nanoformulation for delivering peptide drugs with diverse administration routes are classified based on Table 1.

Parenteral injections: Therapeutic peptides are conventionally formulated as solution or suspension and are administered via subcutaneous injection. Inherently poor physicochemical properties of therapeutic peptides in the gastrointestinal tract, and also low permeability across biological membrane implies the advantages of intravenous injection. The need for repeated administration as a result of short plasma half-life of peptide drugs combined with the risk of infection are disadvantages of this approach. Encapsulating peptides in the nanoformulations with the aim of protection from degradation, as well as conjugation of peptide to the inert polymers, are some of the promising approaches to enhance the stability of peptide drugs.

Oral route: As an alternative to the invasive parenteral route, noninvasive oral administration for peptide drugs is greatly preferred thanks to the low infection risk and high patient compliance. Nevertheless, delivering enough functional peptides in their native form through the oral pathway is a significant unmet challenge. Peptide degradation is ascribed to the acidic environment and proteolytic enzymes in the GI tract and is the main limitation for delivery through this pathway ${ }^{166}$. Low permeability across the GI epithelia also causes an overall poor bioavailability of peptide and protein drugs. Using smart nanoparticles that can protect encapsulated peptides toward enzymatic digestions and releasing them when required have been explored extensively ${ }^{167}$.

Ocular route: The sensitive and complex environment of the eye and poor membrane permeability across ocular barriers and tissues are the main challenges to deliver peptide drugs through the ocular pathway. Two main factors in transcorneal penetration of biological molecules are their size and efficient interaction with the ocular surface. Various nanoparticles alone or in combination with other physical treatments improved the topical passage of proteins and peptide-based drugs through the barriers of ocular system ${ }^{168}$. Nanoparticles can adhere to the ocular surface and increase the contact time of administrated drugs with eye tissue. Furthermore, nanoformulations permit the penetration of drugs to the inner layer of eye structure using a transmucosal route ${ }^{169}$. Controlling the surface charge also is another critical feature of nanoparticles, which has been very useful in ocular delivery ${ }^{170}$.

Respiratory route: Inhalation delivery of therapeutics is a more convenient method in the treatment of respiratory disease. Nevertheless, a large absorptive surface area and high vascularity of endothelial basement membrane of the nasal epithelium make respiratory routes promising for the systemic delivery of active pharmaceutical peptides ${ }^{171}$. Particularly, the respiratory route is favorable for the targeted delivery of drugs to the brain. However, the absorption of therapeutic peptides across airway epithelial cells is strongly limited by clearance mechanisms in the airway ${ }^{172}$. Small nanoparticle sizes conferred the ability to deposit in deep lungs and adhere to the mucosal membrane of respiratory tracts which highly increase their endocytosis by airways epithelial cells ${ }^{173}$. Penetration to the nasal epithelium via extracellular routes is possible for nanoparticles smaller than 50 $\mathrm{nm}$.

In comparison with other airway delivered therapeutics which have larger sizes, nanoparticles are less susceptible to phagocytosis by macrophages. Controlling the surface charge, shape, and hydrophobicity of the nanoparticles can modulate their residence time in the respiratory tract and protect them against clearance by immune system ${ }^{174}$.

Transdermal route: The most significant advantages of the transdermal route are considerably reduced enzymatic activity and avoidance from hepatic first-pass metabolism after administration $^{175}$. To date, most of the delivery approaches based on the transdermal route include small lipophilic drug molecules.

Recently, nanoparticles have been shown to significantly improve the penetration of peptide-based drugs through the stratum corneum layer ${ }^{176}$. The molecular mass, size, and surface charge of particles are also determinative factors in the selection of administration routes ${ }^{177}$. The successful delivery of peptide-based medicines to the target region requires not only an ideal nanocarrier but also an effective strategy that enables carriers to cross the biological barriers after administration. 
Table 1. Nanoformulation examples used for therapeutic peptide delivery

\begin{tabular}{|c|c|c|c|c|c|c|c|}
\hline & NPs formulations & Size $(n m)$ & Therapeutic peptide & Peptide length(aa) & $\begin{array}{l}\text { Administratic } \\
\text { routes }\end{array}$ & & $\begin{array}{l}\text { Refer- } \\
\text { ences }\end{array}$ \\
\hline \multirow{8}{*}{ 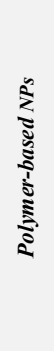 } & \multirow{8}{*}{$\begin{array}{l}\text { HCO-40/OC-40 } \\
\text { PEG-PCL-g-PEI } \\
\text { LMWP-PEG-PLGA } \\
\text { CS/CMS complexes } \\
\text { mPEG-g-alginic acid } \\
\text { Gelatin NPs, Glyco } \\
\text { chitosan }\end{array}$} & $16-20$ & Octreotide & 8 & & 53,178 & \\
\hline & & 22.4 & CsA & 11 & Ocular & 179 & \\
\hline & & 28 & CsA & 11 & Ocular & 40 & \\
\hline & & 114.4 & Exenatide & 39 & Oral & 180 & \\
\hline & & 149 & & & & 181 & \\
\hline & & & Insulin & 51 & Oral & 182183 & \\
\hline & & 72 & Calcitonin & 32 & Oral & & \\
\hline & & $230-330$ & Calcitonin & 32 & Respiratory & & \\
\hline \multirow{6}{*}{ 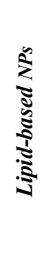 } & Liposome & 322 & Glycoprotein B, Poly lysine & 13 & Ocular & 184 & \\
\hline & & & & & & 74 & \\
\hline & Liposome & $114-157$ & sCT & 32 & Respiratory & 76 & \\
\hline & Liposome & 900 & muramyl tripeptide-PE & 3 & Parenteral & 93,185 & \\
\hline & SLNs & $150-170$ & Insulin and glargine insulin & 51 & Oral & 186 & \\
\hline & SLNs & 200 & CsA & 11 & Transdermal & & \\
\hline \multirow{4}{*}{ 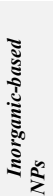 } & & & & & & 120 & \\
\hline & GNPs & 5.4 & Insulin & 51 & Oral & 130 & \\
\hline & $\mathrm{Fe}_{3}-{ }_{8} \mathrm{O}_{4} \mathrm{IONP}$ & 22 & Vancomycin & 7 & Oral & 13 & \\
\hline & MSNs & 50 & Insulin & 51 & $\begin{array}{c}\text { Oral } \\
\text { Respira- }\end{array}$ & & \\
\hline \multirow{4}{*}{ 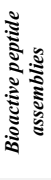 } & DAEP-C16Y & 30 & $\mathrm{C} 16 \mathrm{Y}$ & 12 & Parenteral & 143 & \\
\hline & & 60 & & & & 145 & \\
\hline & PEG-DAEP-T4 & & 14 & 7 & Parenteral & 144 & \\
\hline & $\begin{array}{l}\text { ROS/MMP-Ac2- } \\
26\end{array}$ & & Ac2-26 & 25 & Parenteral & & \\
\hline \multirow{5}{*}{ 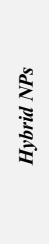 } & \multirow[b]{3}{*}{ SLN associated CS } & & & & & 187 & \\
\hline & & 51 & CsA & 11 & Ocular & 188 & \\
\hline & & 311 & Insulin & 51 & Oral & 189 & \\
\hline & $\begin{array}{l}\text { Polymeric nano- } \\
\text { composite }\end{array}$ & 268 & Salmon calcitonin & 32 & Respiratory & & \\
\hline & $\begin{array}{l}\text { Pharmatose } 325 \mathrm{M}^{\mathrm{TM}} \\
\text { coated PLGA }\end{array}$ & & & & & & \\
\hline
\end{tabular}

CS: chitosan; CMS carboxymethyl chitosan; GNP: gold nanoparticles; MSN: mesoporous silica nanoparticles; SLN: solid lipid nanoparticles.

Conclusion and future perspective

To address unmet needs of medical treatment, the use of peptides as therapeutics has shown tremendous potential over other medications such as proteins, owing to their unique advantages, including high selectivity and biocompatibility. Furthermore, peptide drugs can endure harsh manufacturing conditions better than protein drugs. Nonetheless, due to the given intrinsic weaknesses, peptides are not 
directly suitable for use as available therapeutics. Suboptimal physicochemical and pharmacokinetic properties of peptide therapeutics can be tackled through taking advantage of nanocarrier-based platforms. Over the past decades, nanoparticles and colloidal systems have successfully been applied both in industry and medicine, and many designs and structures have been evaluated. As drug delivery vehicles, nanocarriers are intended to protect peptide payloads from premature degradation, enhance the controlled release of them in the target tissue, and improve their cellular uptake. Synergism between peptide drugs and nanoparticulate systems would make substantial progress toward the development of the next generation of personalized medicine and help to overcome insurmountable challenges in the clinical translations of therapeutic peptides.

The rational design of peptide-carrying nanoparticles should undergo profound adjustments concerning sophisticated physicochemical features of biological systems and target tissues. Delivering pharmacologically active peptide to specific tissues or cells through their incorporation into nanocarriers encounters various challenges. Functional modifications to overcome their drawbacks are under investigation and include hybridization of two or more materials into the unified construction, as well as developing new formulations containing self-assembled peptide nanoparticulates or colloidal systems. Such strategies have the potential to develop advanced peptide-based therapeutics. The need for future research may be centered on the perfection of the safety and efficiency of nanoparticles for noninvasive administration and high patient compliance. Interaction among different research areas such as materials, chemistry, biology, and medicine will enable us to rationally engineer and provide the multifaceted tools to overcome the major challenges that prevent this technology from being translated to the clinic.

Acknowledgments

This work was supported by grants from the National Key R\&D Program of China (2018YFA0208900). We also thank the research core of Tarbiat Modares University for the financial support with grant number IG-39707.

Conflict of interest

The authors declare there is no conflict of interest. For signed statements, please contact the journal office: editor@precisionnanomedicine.com

Quote this article as Atabakhshi-Kashi M, Geranpayehvaghei M, Wang Y, Akhbariyoon H, Taleb M, Zhang Y, Khajeh K, Nie G, Recent Advances of Nanocarriers for Effective Delivery of Therapeutic Peptides, Precis. Nanomed. 2020 July;3(3):549-576, https://doi.org/10.33218/001c.13444

References

1. Rastogi, S., Shukla, S., Kalaivani, M. \& Singh, G. N. Peptide-based therapeutics: quality specifications, regulatory considerations, and prospects. Drug Discov. Today 24, 148-162 (2019).

2. Schlueter, N. et al. Metabolic actions of natriuretic peptides and therapeutic potential in the metabolic syndrome. Pharmacol. Ther. 144, 12-27 (2014).

3. Xiao, Y.-F. et al. Peptide-Based Treatment: A Promising Cancer Therapy. J. Immunol. Res. 2015, 1-13 (2015).

4. Kotraiah, V. et al. Novel Peptide-Based PD1 Immunomodulators Demonstrate Efficacy in Infectious Disease Vaccines and Therapeutics. Front. Immunol. 11, 1-17 (2020).

5. Usmani, S. S. et al. THPdb: Database of FDA-approved peptide and protein therapeutics. PLoS One 12, 1-12 (2017).

6. Research, G. V. Peptide Therapeutics Market By Application (Cancer, Cardiovascular Disorder, Metabolic Disorder, Respiratory Disorder, Pain, Dermatology), By Type (Generic, Innovative) By Type of Manufacturers (In-house, Outsourced), And Segment Forecasts, 2018 - 2025. Peptide Therapeutics Market Size, Growth | Global Industry Report 2025 (2017).

7. Market Research Report, PNS Pharma. Glob. Pept. Ther. Mark. Clin. Trials Insight 2026 (2020).

8. Lee, A. C. L., Harris, J. L., Khanna, K. K. \& Hong, J. H. A comprehensive review on current advances in peptide drug development and design. Int. J. Mol. Sci. 20, 1-21 (2019). 
9. Agyei, D., Ahmed, I., Akram, Z., M. N. Iqbal, H. \& K. Danquah, M. Protein and Peptide Biopharmaceuticals: An Overview. Protein Pept. Lett. 24, 94-101 (2017).

10. Fernández de Ullivarri, M., Arbulu, S., Garcia-Gutierrez, E. \& Cotter, P. D. Antifungal Peptides as Therapeutic Agents. Frontiers in Cellular and Infection Microbiology vol. 10 (2020).

11. Lau, J. L. \& Dunn, M. K. Therapeutic peptides: Historical perspectives, current development trends, and future directions. Bioorganic Med. Chem. 26, 2700-2707 (2018).

12. Keservani, R. K., Sharma, A. K. \& Jarouliya, U. Protein and peptide in drug targeting and its therapeutic approach. Ars Pharm. 56, 165-177 (2015).

13. Agyei, D., Tan, K. X., Pan, S., Udenigwe, C. C. \& Danquah, M. K. Peptides for biopharmaceutical applications. in Woodhead 231-251 (Elsevier Ltd, 2018).

14. Eichler, J. \& Contini, A. Folded synthetic peptides for biomedical applications. Front. Chem. 7, 1-2 (2019).

15. Tesauro, D. et al. Peptide-based drug-delivery systems in biotechnological applications: Recent advances and perspectives. Molecules 24, 1-27 (2019).

16. Uhlig, T. et al. The emergence of peptides in the pharmaceutical business: From exploration to exploitation. EuPA Open Proteomics 4, 58-69 (2014).

17. Wu, L. Regulatory Considerations for Peptide Therapeutics. in RSC Drug Discovery Series vols 2019-Janua 1-30 (2019).

18. Mathieu Verbeken, S. V. D. Purity profiling of Peptide Drugs. J. Bioanal. Biomed. s6, 1-18 (2012).

19. Marqus, S., Pirogova, E. \& Piva, T. J. Evaluation of the use of therapeutic peptides for cancer treatment. J. Biomed. Sci. 24, 1-15 (2017).

20. Jain, D., Mahammad, S. S., Singh, P. P. \& Kodipyaka, R. A review on parenteral delivery of peptides and proteins. Drug Dev. Ind. Pharm. 45, 1403-1420 (2019).

21. Pudlarz, A. \& Szemraj, J. Nanoparticles as carriers of proteins, peptides and other therapeutic molecules. Open Life Sci. 13, 285-298 (2018).

22. Craik, D. J., Fairlie, D. P., Liras, S. \& Price, D. The Future of Peptide-based Drugs. Chem. Biol. Drug Des. 81, 136-147 (2013).

23. Tsai, P. K. et al. Formulation Design of Acidic Fibroblast Growth Factor. Pharm. Res. An Off. J. Am. Assoc. Pharm. Sci. 10, 649-659 (1993).

24. Johnson, O. L. et al. A month-long effect from a single injection of microencapsulated human growth hormone. Nat. Med. 2, 795-799 (1996).

25. Semalty, A., Semalty, M., Singh, R., Saraf, S. \& Saraf, S. Properties and formulation of oral drug delivery systems of protein and peptides. Indian J. Pharm. Sci. 69, 741-747 (2007).

26. Burnham, N. L. Polymers for delivering peptides and proteins. Am. J. Hosp. Pharm. 51, 210218 (1994).

27. Goodwin, D., Simerska, P. \& Toth, I. Peptides As Therapeutics with Enhanced Bioactivity. Curr. Med. Chem. 19, 4451-4461 (2012).

28. Knauer, N., Pashkina, E. \& Apartsin, E. Topological aspects of the design of nanocarriers for therapeutic peptides and proteins. Pharmaceutics 11, (2019).

29. Patra, J. K. et al. Nano based drug delivery systems: recent developments and future prospects. J. Nanobiotechnology 16, 71 (2018).

30. Jain, A. et al. Peptide and protein delivery using new drug delivery systems. Crit. Rev. Ther. Drug Carrier Syst. 30, 293-329 (2013).

31. Jeong, W. jin et al. Peptide-nanoparticle conjugates: a next generation of diagnostic and therapeutic platforms? Nano Converg. 5, 1-18 (2018).

32. Mura, S., Nicolas, J. \& Couvreur, P. Stimuli-responsive nanocarriers for drug delivery. Nat. Mater. 12, 991-1003 (2013).

33. Qin, H., Ding, Y., Mujeeb, A., Zhao, Y. \& Nie, G. Tumor Microenvironment Targeting and Responsive Peptide-Based Nanoformulations for Improved Tumor Therapy. Mol. Pharmacol. 92, 219 231 (2017). 
34. Zaman, R., Othman, I. \& Chowdhury, E. Carrier Mediated Systemic Delivery of Protein and Peptide Therapeutics. Curr. Pharm. Des. 22, 6167-6191 (2016).

35. Brancolini, G., Bellucci, L., Maschio, M. C., Di Felice, R. \& Corni, S. The interaction of peptides and proteins with nanostructures surfaces: a challenge for nanoscience. Curr. Opin. Colloid Interface Sci. 41, 86-94 (2019).

36. Yadav, H. K. S., Almokdad, A. A., Shaluf, S. I. M. \& Debe, M. S. Polymer-Based Nanomaterials for Drug-Delivery Carriers. in Nanocarriers for Drug Delivery 531-556 (Elsevier Inc., 2019).

37. Ostróżka-Cieślik, A. \& Sarecka-Hujar, B. The Use of Nanotechnology in Modern Pharmacotherapy. in Multifunctional Systems for Combined Delivery, Biosensing and Diagnostics 139-158 (Elsevier, 2017).

38. Damgé, C., Michel, C., Aprahamian, M., Couvreur, P. \& Devissaguet, J. P. Nanocapsules as carriers for oral peptide delivery. J. Control. Release 13, 233-239 (1990).

39. Vinothini, K. \& Rajan, M. Mechanism for the nano-based drug delivery system. in Characterization and Biology of Nanomaterials for Drug Delivery 219-263 (Elsevier, 2019). doi:10.1016/B978-0-12-814031-4.00009-X.

40. Zhang, L. et al. The use of low molecular weight protamine to enhance oral absorption of exenatide. Int. J. Pharm. 547, 265-273 (2018).

41. Perinelli, D. R., Cespi, M., Bonacucina, G. \& Palmieri, G. F. PEGylated polylactide (PLA) and poly (lactic-co-glycolic acid) (PLGA) copolymers for the design of drug delivery systems. J. Pharm. Investig. 49, 443-458 (2019).

42. Varshosaz, J., Minaiyan, M. \& Forghanian, M. Prolonged Hypocalcemic Effect by Pulmonary Delivery of Calcitonin Loaded Poly(Methyl Vinyl Ether Maleic Acid) Bioadhesive Nanoparticles. Biomed Res. Int. 2014, 1-13 (2014).

43. Wong, C. Y., Al-Salami, H. \& Dass, C. R. The role of chitosan on oral delivery of peptideloaded nanoparticle formulation. J. Drug Target. 26, 551-562 (2018).

44. Jie, J., Liu, D. \& Zou, X. A one-pot synthesis of hydrophilic poly(glycerol methacrylate) chitosan for highly selective enrichment of glycopeptides. Chem. Commun. 56, 908-911 (2020).

45. Marques, C., Som, C., Schmutz, M., Borges, O. \& Borchard, G. How the Lack of Chitosan Characterization Precludes Implementation of the Safe-by-Design Concept. Front. Bioeng. Biotechnol. 8, 1-12 (2020).

46. Wang, J. et al. Mechanism of surface charge triggered intestinal epithelial tight junction opening upon chitosan nanoparticles for insulin oral delivery. Carbohydr. Polym. (2016) doi:10.1016/j.carbpol.2016.10.021.

47. Lang, X., Wang, T., Sun, M., Chen, X. \& Liu, Y. Advances and applications of chitosanbased nanomaterials as oral delivery carriers: A review. Int. J. Biol. Macromol. 154, 433-445 (2020).

48. Mumuni, M. A., Kenechukwu, F. C., Ofokansi, K. C., Attama, A. A. \& Díaz, D. D. Insulinloaded mucoadhesive nanoparticles based on mucin-chitosan complexes for oral delivery and diabetes treatment. Carbohydr. Polym. 229, 115506 (2020).

49. Blachman, A. et al. Targeted anti-inflammatory peptide delivery in injured endothelial cells using dermatan sulfate/chitosan nanomaterials. Carbohydr. Polym. 230, 115610 (2020).

50. Mandal, A. et al. Ocular delivery of proteins and peptides: Challenges and novel formulation approaches. Adv. Drug Deliv. Rev. 126, 67-95 (2018).

51. Saricaoğlu, H., Yazici, S., Zorlu, Ö., Bülbül Başkan, E. \& Aydoğan, K. Cyclosporine-A for severe childhood atopic dermatitis: Clinical experience on efficacy and safety profile. Turkish J. Med. Sci. 48, 933-938 (2018).

52. Yenice, I. et al. Hyaluronic acid coated poly- $\varepsilon$-caprolactone nanospheres deliver high concentrations of cyclosporine A into the cornea. Exp. Eye Res. 87, 162-167 (2008).

53. Mandal, A., Patel, P., Pal, D. \& Mitra, A. K. Multi-Layered Nanomicelles as Self-Assembled Nanocarrier Systems for Ocular Peptide Delivery. AAPS PharmSciTech 20, 1-17 (2019).

54. Yasmeen, Mamatha, T., Zubair, M., Begum, S. \& Muneera, T. Various emerging trends in insulin drug delivery systems. Br. J. Pharm. Res. 5, 294-308 (2015). 
55. Liu, J. et al. Effect of inner $\mathrm{pH}$ on peptide acylation within PLGA microspheres. Eur. J. Pharm. Sci. 134, 69-80 (2019).

56. Zhang, Y. \& Schwendeman, S. P. Minimizing acylation of peptides in PLGA microspheres. J. Control. Release 162, 119-126 (2012).

57. Park, H. et al. Effect of Stabilizers on Encapsulation Efficiency and Release Behavior of Exenatide-Loaded PLGA Microsphere Prepared by the W/O/W Solvent Evaporation Method. Pharmaceutics 11, 627 (2019)

58. Izawa, H., Kinai, M., Ifuku, S., Morimoto, M. \& Saimoto, H. Guanidinylated chitosan inspired by arginine-rich cell-penetrating peptides. Int. J. Biol. Macromol. 125, 901-905 (2019).

59. Lollo, G. et al. Polyarginine Nanocapsules as a Potential Oral Peptide Delivery Carrier. J. Pharm. Sci. 106, 611-618 (2017)

60. Güncüm, E. et al. Development and characterization of polymeric-based nanoparticles for sustained release of amoxicillin-an antimicrobial drug. Artif. Cells, Nanomedicine Biotechnol. 46, 964 973 (2018).

61. Doostmohammadi, M. et al. Hydrogels For Peptide Hormones Delivery: Therapeutic And Tissue Engineering Applications. Drug Des. Devel. Ther. 13, 3405-3418 (2019).

62. Seo, B. B., Park, M. R. \& Song, S. C. Sustained Release of Exendin 4 Using Injectable and Ionic-Nano-Complex Forming Polymer Hydrogel System for Long-Term Treatment of Type 2 Diabetes Mellitus. ACS Appl. Mater. Interfaces 11, 15201-15211 (2019).

63. Balmert, S. C. et al. Positive charge of 'sticky' peptides and proteins impedes release from negatively charged PLGA matrices. J. Mater. Chem. B 3, 4723-4734 (2015).

64. Souery, W. N. et al. Controlling and quantifying the stability of amino acid-based cargo within polymeric delivery systems. J. Control. Release 300, 102-113 (2019).

65. Paliwal, R., Babu, R. J. \& Palakurthi, S. Nanomedicine Scale-up Technologies: Feasibilities and Challenges. Ageing Int. 15, 1527-1534 (2014).

66. Bovone, G., Steiner, F., Guzzi, E. A. \& Tibbitt, M. W. Automated and Continuous Production of Polymeric Nanoparticles. Front. Bioeng. Biotechnol. 7, 1-11 (2019).

67. Belcher, D. A., Cuddington, C. T., Martindale, E. L., Pires, I. S. \& Palmer, A. F. Controlled Polymerization and Ultrafiltration Increase the Consistency of Polymerized Hemoglobin for Use as an Oxygen Carrier. Bioconjug. Chem. 31, 605-621 (2020).

68. Knox, S. T. \& Warren, N. J. Enabling technologies in polymer synthesis: Accessing a new design space for advanced polymer materials. React. Chem. Eng. 5, 405-423 (2020).

69. Calzoni, E. et al. Biocompatible polymer nanoparticles for drug delivery applications in cancer and neurodegenerative disorder therapies. J. Funct. Biomater. 10, 1-15 (2019).

70. Alavi, M., Karimi, N. \& Safaei, M. Application of various types of liposomes in drug delivery systems. Adv. Pharm. Bull. 7, 3-9 (2017).

71. Sun, H. et al. A multifunctional liposomal nanoplatform co-delivering hydrophobic and hydrophilic doxorubicin for complete eradication of xenografted tumors. Nanoscale 11, 17759-17772 (2019).

72. Panahi, Y. et al. Recent advances on liposomal nanoparticles: synthesis, characterization and biomedical applications. Artif. Cells, Nanomedicine Biotechnol. 45, 788-799 (2017).

73. Gracia, B. et al. Nanomedicine review : clinical developments in liposomal applications. Cancer Nanotechnology (Springer Vienna, 2019).

74. Chen, M. et al. Improved absorption of salmon calcitonin by ultraflexible liposomes through intranasal delivery. Peptides 30, 1288-1295 (2009).

75. Behr, J. et al. Lung deposition of a liposomal cyclosporine a inhalation solution in patients after lung transplantation. J. Aerosol Med. Pulm. Drug Deliv. 22, 121-129 (2009).

76. Biteau, K. et al. L-MTP-PE and zoledronic acid combination in osteosarcoma: Preclinical evidence of positive therapeutic combination for clinical transfer. Am. J. Cancer Res. 6, 677-689 (2016).

77. Meyers, P. A. Muramyl tripeptide (mifamurtide) for the treatment of osteosarcoma. Expert Rev. Anticancer Ther. 9, 1035-1049 (2009). 
78. Bulbake, U., Doppalapudi, S., Kommineni, N. \& Khan, W. Liposomal formulations in clinical use: An updated review. Pharmaceutics 9, 1-33 (2017).

79. Kroemer, G., Zitvogel, L. \& Galluzzi, L. Victories and deceptions in tumor immunology: Stimuvax ${ }^{\circledR}$. Oncoimmunology 2, 1-3 (2013).

80. Vijayan, V., Mohapatra, A., Uthaman, S. \& Park, I. K. Recent advances in nanovaccines using biomimetic immunomodulatory materials. Pharmaceutics 11, 1-27 (2019).

81. Rezaei, N. et al. Encapsulation of an endostatin peptide in liposomes: Stability, release, and cytotoxicity study. Colloids Surfaces B Biointerfaces 185, 110552 (2020).

82. Donnenfeld, E. D. et al. Lifitegrast for the treatment of dry eye disease in adults. Expert Opin. Pharmacother. 18, 1517-1524 (2017).

83. Lallemand, F., Daull, P., Benita, S., Buggage, R. \& Garrigue, J. Successfully Improving Ocular Drug Delivery Using the Cationic Nanoemulsion, Novasorb. J. Drug Deliv. 2012, 1-16 (2012).

84. Bunney, P. E., Zink, A. N., Holm, A. A., Billington, C. J. \& Kotz, C. M. A comprehensive insight on ocular pharmacokinetics. Physiol. Behav. 176, 139-148 (2017).

85. NCT00089180. T4N5 Liposomal Lotion in Preventing The Recurrence of Nonmelanoma Skin Cancer in Patients Who Have Undergone a Kidney Transplant( ClinicalTrials). ClinicalTrials.gov.

86. Lyons, R. M., Wilks, S. T., Young, S. \& Brown, G. L. Oral ezatiostat HCl (Telintra, TLK199) and idiopathic chronic neutropenia (ICN): A case report of complete response of a patient with GCSF resistant ICN following treatment with ezatiostat, a glutathione S-transferase P1-1 (GSTP1-1) inhibitor. J. Hematol. Oncol. 4, 43 (2011).

87. Tiwari, R., Pandey, V., Asati, S., Soni, V. \& Jain, D. Therapeutic challenges in ocular delivery of lipid based emulsion. Egypt. J. Basic Appl. Sci. 5, 121-129 (2018).

88. Lallemand, F. et al. Cyclosporine A delivery to the eye: A comprehensive review of academic and industrial efforts. Eur. J. Pharm. Biopharm. 117, 14-28 (2017).

89. Mu, H. \& Holm, R. Solid lipid nanocarriers in drug delivery: characterization and design. Expert Opin. Drug Deliv. 15, 771-785 (2018).

90. He, H. et al. Solid lipid nanoparticles as a drug delivery system to across the blood-brain barrier. Biochem. Biophys. Res. Commun. 519, 385-390 (2019).

91. Aldawsari, H. M. \& Hosny, K. M. Solid lipid nanoparticles of Vancomycin loaded with Ellagic acid as a tool for overcoming nephrotoxic side effects: Preparation, characterization, and nephrotoxicity evaluation. J. Drug Deliv. Sci. Technol. 45, 76-80 (2018).

92. Gandomi, N. et al. Solid lipid nanoparticles surface modified with anti-Contactin-2 or antiNeurofascin for brain-targeted delivery of medicines. Pharm. Dev. Technol. 22, 426-435 (2017).

93. $\mathrm{Xu}, \mathrm{Y}$. et al. Novel Solid Lipid Nanoparticle with Endosomal Escape Function for Oral Delivery of Insulin. ACS Appl. Mater. Interfaces 10, 9315-9324 (2018).

94. Lai, K. K. et al. Identification of Novel Fusion Inhibitors of Influenza A Virus by Chemical Genetics. J. Virol. 90, 2690-2701 (2016).

95. Alsulays, B. B., Anwer, M. K., Soliman, G. A., Alshehri, S. M. \& Khafagy, E.-S. Impact Of Penetratin Stereochemistry On The Oral Bioavailability Of Insulin-Loaded Solid Lipid Nanoparticles. Int. J. Nanomedicine 14, 9127-9138 (2019).

96. Ghasemiyeh, P. \& Mohammadi-Samani, S. Solid lipid nanoparticles and nanostructured lipid carriers as novel drug delivery systems: Applications, advantages and disadvantages. Res. Pharm. Sci. 13, 288-303 (2018).

97. Ahmad, J. et al. Bile Salt Stabilized Vesicles (Bilosomes): A Novel Nano-Pharmaceutical Design for Oral Delivery of Proteins and Peptides. Curr. Pharm. Des. 23, 1575-1588 (2017).

98. Dave, V., Tak, K., Sohgaura, A., Gupta, A. \& Sadhu, V. Lipid-polymer hybrid nanoparticles : Synthesis strategies and biomedical applications. J. Microbiol. Methods 160, 130-142 (2019).

99. Cai, J. et al. A core-shell polymeric-inorganic hybrid nanocomposite system for MRI-visible gene delivery application in cancer immunotherapy. J. Ind. Eng. Chem. 76, 188-196 (2019).

100. Babaie, S., Del Bakhshayesh, A. R., Ha, J. W., Hamishehkar, H. \& Kim, K. H. Invasome: A novel nanocarrier for transdermal drug delivery. Nanomaterials 10, 1-12 (2020). 
101. Jiang, T. et al. Enhanced Transdermal Drug Delivery by Transfersome-Embedded Oligopeptide Hydrogel for Topical Chemotherapy of Melanoma. ACS Nano 12, 9693-9701 (2018).

102. Kumar, A. Transferosome: a recent approach for transdermal drug delivery. J. Drug Deliv. Ther. 8, 100-104 (2018).

103. Scaletti, F. et al. Protein delivery into cells using inorganic nanoparticle-protein supramolecular assemblies. Chemical Society Reviews 47, 3421-3432 (2018).

104. Giner-Casares, J. J., Henriksen-Lacey, M., Coronado-Puchau, M. \& Liz-Marzán, L. M. Inorganic nanoparticles for biomedicine: Where materials scientists meet medical research. Mater. Today 19, 19-28 (2016).

105. Raju, G., Katiyar, N., Vadukumpully, S. \& Shankarappa, S. A. Penetration of gold nanoparticles across the stratum corneum layer of. J. Dermatol. Sci. 89, 146-154 (2018).

106. Malmsten, M. Inorganic nanomaterials as delivery systems for proteins, peptides, DNA, and siRNA. Curr. Opin. Colloid Interface Sci. 18, 468-480 (2013).

107. Foroozandeh, P. \& Aziz, A. A. Insight into Cellular Uptake and Intracellular Trafficking of Nanoparticles. Nanoscale Res. Lett. 13, 339 (2018).

108. Daneshjou, S., Dabirmanesh, B., Rahimi, F. \& Khajeh, K. Porous silicon nanoparticle as a stabilizing support for chondroitinase. Int. J. Biol. Macromol. 94, 852-858 (2017).

109. Braun, K. et al. Membrane interactions of mesoporous silica nanoparticles as carriers of antimicrobial peptides. J. Colloid Interface Sci. 475, 161-170 (2016).

110. Tenland, E. et al. Effective delivery of the anti-mycobacterial peptide NZX in mesoporous silica nanoparticles. PLoS One 14, 1-16 (2019).

111. Zhou, Y. et al. Mesoporous silica nanoparticles for drug and gene delivery. Acta Pharm. Sin. B $8,165-177$ (2018).

112. Izquierdo-Barba, I. et al. Incorporation of antimicrobial compounds in mesoporous silica film monolith. Biomaterials 30, 5729-5736 (2009).

113. Kalimuthu, K. et al. Gold nanoparticles stabilize peptide-drug-conjugates for sustained targeted drug delivery to cancer cells. J. Nanobiotechnology 16, 1-13 (2018).

114. Zong, J., Cobb, S. L. \& Cameron, N. R. Peptide-functionalized gold nanoparticles: Versatile biomaterials for diagnostic and therapeutic applications. Biomater. Sci. 5, 872-886 (2017).

115. Hosta-Rigau, L. et al. Multifunctionalized gold nanoparticles with peptides targeted to gastrinreleasing peptide receptor of a tumor cell line. Bioconjug. Chem. 21, 1070-1078 (2010).

116. Kumar, A. et al. Gold nanoparticles functionalized with therapeutic and targeted peptides for cancer treatment. Biomaterials 33, 1180-1189 (2012).

117. Matea, C. T., Mocan, T., Tabaran, F., Iancu, C. \& Mocan, L. C. Rational design of gold nanocarrier for the delivery of JAG-1 peptide. J. Nanobiotechnology 13, 1-10 (2015).

118. Shilo, M. et al. Insulin-coated gold nanoparticles as a new concept for personalized and adjustable glucose regulation. Nanoscale 7, 20489-20496 (2015).

119. Devi, C., Kalita, P., Choudhury, D. \& Barthakur, M. Preparation and characterization of gold nanoparticles conjugated insulin. in Smart Healthcare for Disease Diagnosis and Prevention, 29-32 (INC, 2020). doi:10.1016/b978-0-12-817913-0.00005-5.

120. Sheridan, C. Proof of concept for next-generation nanoparticle drugs in humans. Nat. Biotechnol. 30, 471-473 (2012).

121. Fosgerau, K. \& Hoffmann, T. Peptide therapeutics: Current status and future directions. Drug Discov. Today 20, 122-128 (2015).

122. Yong, K. T. et al. Preparation of quantum dot/drug nanoparticle formulations for traceable targeted delivery and therapy. Theranostics 2, 681-694 (2012).

123. Carrillo-Carrion, C., Atabakhshi-Kashi, M., Carril, M., Khajeh, K. \& Parak, W. J. Taking Advantage of Hydrophobic Fluorine Interactions for Self-Assembled Quantum Dots as a Delivery Platform for Enzymes. Angew. Chemie - Int. Ed. 57, 5033-5036 (2018). 
124. Mohid, S. A. et al. Application of tungsten disulfide quantum dot-conjugated antimicrobial peptides in bio-imaging and antimicrobial therapy. Colloids Surfaces B Biointerfaces 176, 360-370 (2019).

125. Ranjbar-Navazi, Z., Omidi, Y., Eskandani, M. \& Davaran, S. Cadmium-free quantum dotbased theranostics. TrAC - Trends Anal. Chem. 118, 386-400 (2019).

126. Sur, A., Pradhan, B., Banerjee, A. \& Aich, P. Immune Activation Efficacy of Indolicidin Is Enhanced upon Conjugation with Carbon Nanotubes and Gold Nanoparticles. PLoS One 10, 1-15 (2015).

127. Villa, C. H. et al. Single-walled carbon nanotubes deliver peptide antigen into dendritic cells and enhance IgG responses to tumor-associated antigens. ACS Nano 5, 5300-5311 (2011).

128. Kostarelos, K., Lacerda, L., Partidos, C. D., Prato, M. \& Bianco, A. Carbon nanotube-mediated delivery of peptides and genes to cells: Translating nanobiotechnology to therapeutics. J. Drug Deliv. Sci. Technol. 15, 41-47 (2005).

129. Lee, W. et al. Octahedron Iron Oxide Nanocrystals Prohibited Clostridium difficile Spore Germination and Attenuated Local and Systemic Inflammation. Sci. Rep. 7, 1-12 (2017).

130. Chen, Y. H. et al. Vancomycin-loaded nanoparticles enhance sporicidal and antibacterial efficacy for clostridium difficile infection. Front. Microbiol. 10, 1-11 (2019).

131. Cherukula, K. et al. Multifunctional inorganic nanoparticles: Recent progress in thermal therapy and imaging. Nanomaterials 6, 1-26 (2016).

132. Nam, J. et al. Surface engineering of inorganic nanoparticles for imaging and therapy. Adv. Drug Deliv. Rev. 65, 622-648 (2013).

133. Korpi, A., Anaya-Plaza, E., Välimäki, S. \& Kostiainen, M. Highly ordered protein cage assemblies: A toolkit for new materials. WIREs Nanomedicine and Nanobiotechnology 12, (2020).

134. Künzle, M., Lach, M. \& Beck, T. Multi-Component Self-Assembly of Proteins and Inorganic Particles: From Discrete Structures to Biomimetic Materials. Isr. J. Chem. 59, 906-912 (2019).

135. Künzle, M., Mangler, J., Lach, M. \& Beck, T. Peptide-directed encapsulation of inorganic nanoparticles into protein containers. Nanoscale 10, 22917-22926 (2018).

136. Jiao, M. et al. Recent advancements in biocompatible inorganic nanoparticles towards biomedical applications. Biomaterials Science 6, 726-745 (2018).

137. Yang, G., Phua, S. Z. F., Bindra, A. K. \& Zhao, Y. Degradability and Clearance of Inorganic Nanoparticles for Biomedical Applications. Advanced Materials 31, 1-23 (2019).

138. Sis, M. J. \& Webber, M. J. Drug Delivery with Designed Peptide Assemblies. Trends Pharmacol. Sci. 40, 747-762 (2019).

139. Lee, S. et al. Self-assembling peptides and their application in the treatment of diseases. Int. J. Mol. Sci. 20, (2019).

140. Edwards-Gayle, C. J. C. \& Hamley, I. W. Self-assembly of bioactive peptides, peptide conjugates, and peptide mimetic materials. Org. Biomol. Chem. 15, 5867-5876 (2017).

141. Ji, T. et al. Peptide Assembly Integration of Fibroblast-Targeting and Cell-Penetration Features for Enhanced Antitumor Drug Delivery. Adv. Mater. 27, 1865-1873 (2015).

142. Zhao, Y. et al. Transformable peptide nanocarriers for expeditious drug release and effective cancer therapy via cancer-associated fibroblast activation. Angew. Chemie - Int. Ed. 55, 1050-1055 (2016)

143. Ding, Y. et al. Improvement of stability and efficacy of $\mathrm{C} 16 \mathrm{Y}$ therapeutic peptide via molecular self-assembly into tumor-responsive nanoformulation. Mol. Cancer Ther. 14, 2390-2400 (2015).

144. Peters, E. B. et al. Atheroma Niche-Responsive Nanocarriers for Immunotherapeutic Delivery. Adv. Healthc. Mater. 8, 1-12 (2019).

145. Zhang, L. et al. Cooperatively Responsive Peptide Nanotherapeutic that Regulates Angiopoietin Receptor Tie2 Activity in Tumor Microenvironment to Prevent Breast Tumor Relapse after Chemotherapy. ACS Nano 13, 5091-5102 (2019).

146. Cheng, K. et al. Sequentially Responsive Therapeutic Peptide Assembling Nanoparticles for Dual-Targeted Cancer Immunotherapy. Nano Lett. 18, 3250-3258 (2018). 
147. Kuzmin, D. V. et al. Comparative in vitro study on cytotoxicity of recombinant $\beta$-hairpin peptides. Chem. Biol. Drug Des. 91, 294-303 (2018).

148. Meroni, D. \& Ardizzone, S. Preparation and application of hybrid nanomaterials. Nanomaterials 8, 2-5 (2018).

149. Moghimi, S. M. \& Szebeni, J. Stealth liposomes and long circulating nanoparticles: Critical issues in pharmacokinetics, opsonization and protein-binding properties. Progress in Lipid Research 42, 463-478 (2003).

150. Cantor, S. et al. Evaluation of the Antimicrobial Activity of Cationic Peptides Loaded in Surface-Modified Nanoliposomes against Foodborne Bacteria. Int. J. Mol. Sci. 20, 680 (2019).

151. da Silva, I. M., Boelter, J. F., da Silveira, N. P. \& Brandelli, A. Phosphatidylcholine nanovesicles coated with chitosan or chondroitin sulfate as novel devices for bacteriocin delivery. J. Nanoparticle Res. 16, 2479 (2014).

152. Lopes, N. A., Pinilla, C. M. B. \& Brandelli, A. Pectin and polygalacturonic acid-coated liposomes as novel delivery system for nisin: Preparation, characterization and release behavior. Food Hydrocoll. 70, 1-7 (2017).

153. Mir, S. H. et al. Organic-Inorganic Hybrid Functional Materials: An Integrated Platform for Applied Technologies. J. Electrochem. Soc. 165, B3137-B3156 (2018).

154. Oshiro Junior, J. et al. Drug Delivery Systems Obtained from Silica Based Organic-Inorganic Hybrids. Polymers (Basel). 8, 91 (2016).

155. Jin, Y. et al. Nanosystem composed with MSNs, gadolinium, liposome and cytotoxic peptides for tumor theranostics. Colloids Surfaces B Biointerfaces 151, 240-248 (2017).

156. Xie, J., Xu, W., Wu, Y., Niu, B. \& Zhang, X. Macroporous organosilicon nanocomposites codeliver Bcl2-converting peptide and chemotherapeutic agent for synergistic treatment against multidrug resistant cancer. Cancer Lett. 469, 340-354 (2020).

157. Mansoor, S., Kondiah, P. P. D., Choonara, Y. E. \& Pillay, V. Polymer-Based Nanoparticle Strategies for Insulin Delivery. Polymers (Basel). 11, 1-27 (2019).

158. Lee, K. C., Chen, W. J. \& Chen, Y. C. Using Dextran-encapsulated gold nanoparticles as insulin carriers to prolong insulin activity. Nanomedicine 12, 1823-1834 (2017).

159. Cho, H.-J. et al. Chondroitin sulfate-capped gold nanoparticles for the oral delivery of insulin. Int. J. Biol. Macromol. 63, 15-20 (2014).

160. Paik, B. A., Mane, S. R., Jia, X. \& Kiick, K. L. Responsive hybrid (poly)peptide-polymer conjugates. J. Mater. Chem. B 5, 8274-8288 (2017).

161. Wilson, P. Synthesis and Applications of Protein/Peptide-Polymer Conjugates. Macromol. Chem. Phys. 218, 1-15 (2017).

162. Cong, Y. et al. Microenvironment-Induced In Situ Self-Assembly of Polymer-Peptide Conjugates That Attack Solid Tumors Deeply. Angew. Chemie - Int. Ed. 58, 4632-4637 (2019).

163. Jia, H. R. et al. Construction of Dually Responsive Nanotransformers with Nanosphere-Nanofiber-Nanosphere Transition for Overcoming the Size Paradox of Anticancer Nanodrugs. ACS Nano 13, 11781-11792 (2019)

164. Tamarov, K., Näkki, S., Xu, W. \& Lehto, V. P. Approaches to improve the biocompatibility and systemic circulation of inorganic porous nanoparticles. Journal of Materials Chemistry B vol. 6 3632-3649 (2018)

165. Kreyling, W. G. et al. In vivo integrity of polymer-coated gold nanoparticles. Nat. Nanotechnol. $10,619-623(2015)$.

166. Cao, S. jun et al. Nanoparticles: Oral Delivery for Protein and Peptide Drugs. AAPS PharmSciTech 20, 1-11 (2019).

167. Zeng, Z. et al. Stimuli-responsive self-assembled dendrimers for oral protein delivery. J. Control. Release 315, 206-213 (2019).

168. Diebold, Y. \& Calonge, M. Applications of nanoparticles in ophthalmology. Prog. Retin. Eye Res. 29, 596-609 (2010). 
169. Mahlumba, P., Choonara, Y., Kumar, P., du Toit, L. \& Pillay, V. Stimuli-Responsive Polymeric Systems for Controlled Protein and Peptide Delivery: Future Implications for Ocular Delivery. Molecules 21, 1002 (2016).

170. Fabiano, A. et al. Impact of Different Mucoadhesive Polymeric Nanoparticles Loaded in Thermosensitive Hydrogels on Transcorneal Administration of 5-Fluorouracil. Pharmaceutics 11, 623 (2019).

171. Borghardt, J. M., Kloft, C. \& Sharma, A. Inhaled Therapy in Respiratory Disease: The Complex Interplay of Pulmonary Kinetic Processes. Can. Respir. J. 2018, 1-11 (2018).

172. Fellner, R. C., Terryah, S. T. \& Tarran, R. Inhaled protein/peptide-based therapies for respiratory disease. Mol. Cell. Pediatr. 3, 3-7 (2016).

173. Kaur, G., Narang, R. K., Rath, G. \& Goyal, A. K. Advances in pulmonary delivery of nanoparticles. Artif. Cells, Blood Substitutes, Biotechnol. 40, 75-96 (2012).

174. Dombu, C. Y. \& Betbeder, D. Airway delivery of peptides and proteins using nanoparticles. Biomaterials 34, 516-525 (2013).

175. Herwadkar, A. \& Banga, A. K. Transdermal delivery of peptides and proteins. in Peptide and Protein Delivery 69-86 (Elsevier, 2011).

176. Zhang, Y. et al. Advances in transdermal insulin delivery. Adv. Drug Deliv. Rev. 139, 51-70 (2019).

177. Chenthamara, D. et al. Therapeutic efficacy of nanoparticles and routes of administration. Biomater. Res. 23, 1-29 (2019).

178. Cholkar, K., Gilger, B. C. \& Mitra, A. K. Topical, Aqueous, Clear Cyclosporine Formulation Design for Anterior and Posterior Ocular Delivery. Transl. Vis. Sci. Technol. 4, 1-16 (2015).

179. Li, J. et al. Positively charged micelles based on a triblock copolymer demonstrate enhanced corneal penetration. Int. J. Nanomedicine 10, 6027-6037 (2015).

180. Wang, J. et al. Mechanism of surface charge triggered intestinal epithelial tight junction opening upon chitosan nanoparticles for insulin oral delivery. Carbohydr. Polym. 157, 596-602 (2017).

181. Li, N. et al. The use of polyion complex micelles to enhance the oral delivery of salmon calcitonin and transport mechanism across the intestinal epithelial barrier. Biomaterials 33, 88818892 (2012).

182. Morimoto, K. et al. Evaluation of gelatin microspheres for nasal and intramuscular administrations of salmon calcitonin. Eur. J. Pharm. Sci. 13, 179-185 (2001).

183. Makhlof, A., Werle, M., Tozuka, Y. \& Takeuchi, H. Nanoparticles of glycol chitosan and its thiolated derivative significantly improved the pulmonary delivery of calcitonin. Int. J. Pharm. 397, 92-95 (2010).

184. Cortesi, R. et al. Cationic liposomes as potential carriers for ocular administration of peptides with anti-herpetic activity. Int. J. Pharm. 317, 90-100 (2006).

185. Almeida, A. J. \& Souto, E. Solid lipid nanoparticles as a drug delivery system for peptides and proteins. Adv. Drug Deliv. Rev. 59, 478-490 (2007).

186. Essaghraoui, A. et al. Improved dermal delivery of cyclosporine a loaded in solid lipid nanoparticles. Nanomaterials 9, 1-14 (2019).

187. Sandri, G. et al. Chitosan-associated SLN: In vitro and ex vivo characterization of cyclosporine A loaded ophthalmic systems. J. Microencapsul. 27, 735-746 (2010).

188. Ji, N. et al. Chitosan coating of zein-carboxymethylated short-chain amylose nanocomposites improves oral bioavailability of insulin in vitro and in vivo. J. Control. Release 313, 1-13 (2019).

189. Yang, M. et al. Design and evaluation of poly(dl-lactic-co-glycolic acid) nanocomposite particles containing salmon calcitonin for inhalation. Eur. J. Pharm. Sci. 46, 374-380 (2012). 